\title{
Unsteady characteristics of S-duct intake flow
}

\section{distortion}

\author{
Daniel Gil-Prieto, Pavlos K. Zachos ${ }^{1}$, David G. MacManus and Grant McLelland \\ Propulsion Engineering Centre, School of Aerospace, Transport and Manufacturing, Cranfield University, \\ Cranfield, MK43 0AL, United Kingdom
}

\begin{abstract}
The unsteady distorted flow fields generated within convoluted intakes can have a detrimental effect on the stability of an aero-engine. The frequency signature in the distorted flow field is of key importance to the engine's response. In this work, time-resolved particle image velocimetry is used to obtain the three-component velocity field at the outlet plane of two S-duct intake configurations for a range of inlet Mach numbers. Proper orthogonal decomposition of the time-resolved velocity data allows the identification of the main frequencies and coherent structures in the flow. The most energetic unsteady structures comprise an in-plane vortex switching mode, associated with a lateral oscillation of the main loss region, and a vertical oscillation of the main loss region. The switching structure occurs at a frequency of $\mathrm{St}=0.42$ and 0.32 for the high and low offset ducts, respectively. The vertical perturbation is associated with a more broadband spectrum between approximately $\mathrm{St}=0.6-1.0$ and $\mathrm{St}=0.26-1.0$ for the high and low offset configurations, respectively. The determined frequencies for these main unsteady flow structures are within the range, which is expected to be detrimental to the operating stability of an aero-engine. The results provide a novel, time-resolved dataset of synchronous velocity measurements of high spatial resolution that enables analysis of the unsteady flows at the exit of complex aero-engine intakes.
\end{abstract}

\footnotetext{
${ }^{1}$ Corresponding author. E-mail: p.zachos@cranfield.ac.uk Phone: +44 (0) 1234754633
} 
Page 2 of 36

\section{Nomenclature}

$A \quad=\quad$ S-duct cross section area, $\mathrm{mm}^{2}$

$A R=$ Area Ratio, $A_{\text {out }} / A_{\text {in }}$

$D=$ S-duct cross section diameter, $\mathrm{mm}$

$E V T=$ Extreme Value Theory

$f \quad=$ Frequency, $\mathrm{Hz}$

$H \quad=\quad$ S-duct centerline offset, $\mathrm{mm}$

$L \quad=\quad$ S-duct axial length, $\mathrm{mm}$

$M \quad=$ Mach number

$R \quad=\quad \mathrm{S}$-duct cross section radius, $\mathrm{mm}$

$R_{c} \quad=$ Curvature radius of the $\mathrm{S}$-duct bend, $\mathrm{mm}$

$\operatorname{Re}_{D}=$ Reynolds number based on the inlet diameter

$s \quad=\quad$ S-duct centerline co-ordinate, $\mathrm{mm}$

$S D=$ Swirl Directivity distortion descriptor

$S I \quad=$ Swirl Intensity distortion descriptor, deg

$S P \quad=\quad$ Swirl Pairs distortion descriptor

$S S_{i, k}^{+}=$Positive Sector Swirl in the k-th swirling region of the i-th ring

$S S_{i, k}^{-}=$Negative Sector Swirl in the k-th swirling region of the i-th ring

$S t \quad=$ Strouhal number, $f D_{A I P} /\langle\bar{w}\rangle_{A I P}$

$T K E=$ Turbulent Kinetic Energy, $\mathrm{J} / \mathrm{kg}$

$u, v, w=$ Velocity vector cartesian components, $\mathrm{m} / \mathrm{s}$

$x, y, z=$ Cartesian coordinate system

$v_{\theta}=$ Circumferential velocity component, $\mathrm{m} / \mathrm{s}$

$\alpha \quad=\quad$ Swirl angle, $\arctan \left(v_{\theta} / w\right), \operatorname{deg}$

$\alpha_{1} \quad=$ Maximum sector swirl descriptor, deg

$\gamma=$ Curvature ratio based on the inlet-section radius, $R_{\text {in }} / R_{c}$

$\theta_{1}=$ Extent of the maximum sector swirl region, deg

$\Phi=$ POD non-dimensional modal distribution 
Page 3 of 36

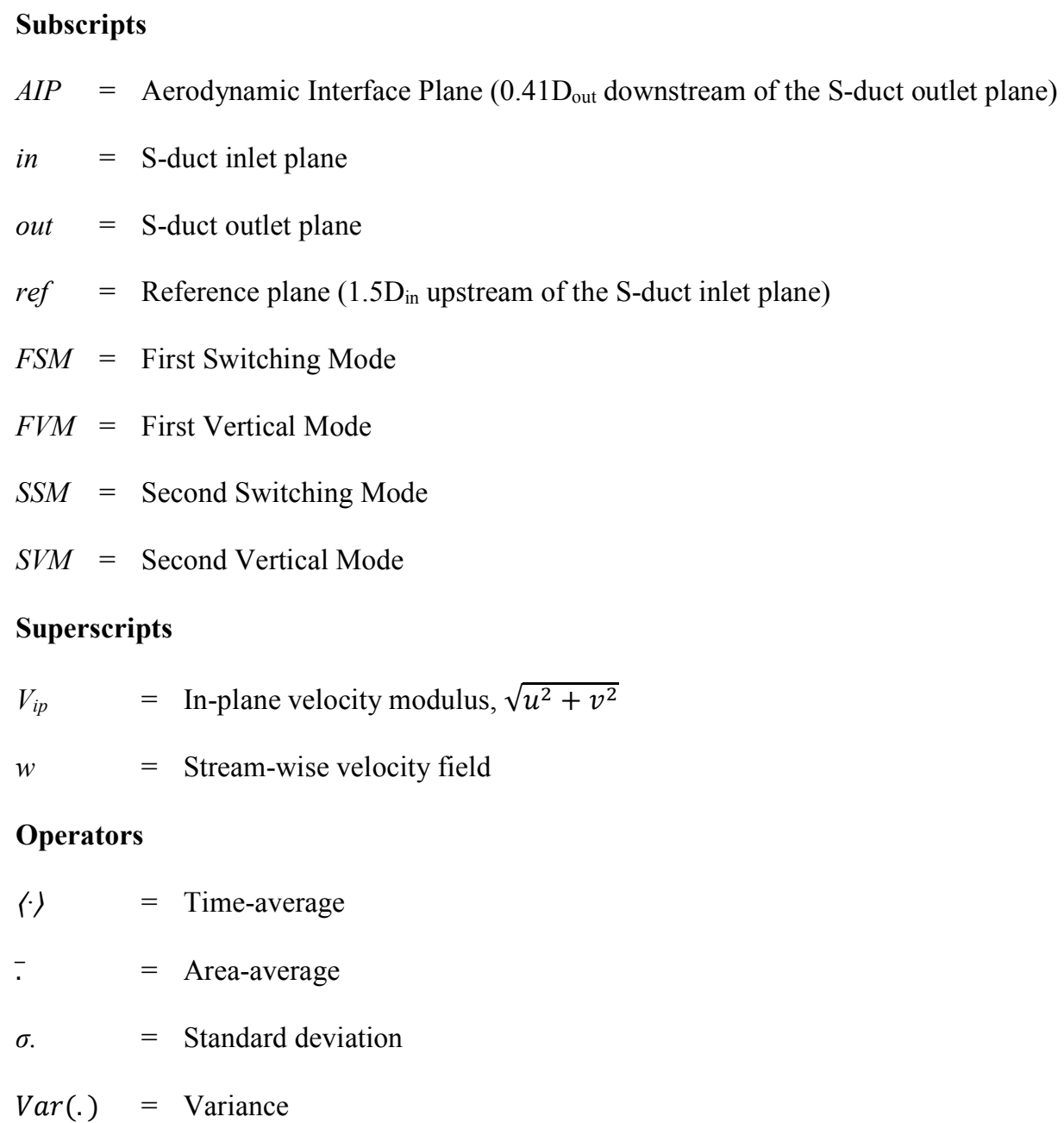

\section{Introduction}

It is expected that future aircraft configurations will require more closely coupled propulsion and airframe architectures (Kawai et al. 2006; Kim and Liou 2013; Florea et al. 2015; Gan and Zhang 2017) which often feature convoluted intakes to deliver the air flow to the engine. Although such aircraft configurations may allow for weight and drag benefits, the coupling between the intake and engine compression system becomes more critical. It is known that complex intakes can generate high levels of unsteady flow distortion, which can reduce the surge margin of the engine and eventually result in engine instabilities (SAE 2007). Historically, flow distortion assessments were based on relatively sparse total pressure measurements acquired using steady, intrusive probes at the Aerodynamic Interface 


\section{Page 4 of 36}

Plane (AIP) ahead of the engine compressor (Wellborn et al. 1993; Toge and Pradeep 2017). For example, the mean flow within convoluted intakes was measured by Wellborn et al. (1993) for a mild geometry $(\mathrm{H} / \mathrm{L}=0.27, \mathrm{AR}=1.52$, $\mathrm{L} / \mathrm{D}_{\text {in }}=5.0$, see Fig. 1), and is characterized by the presence of a main loss region at the lower sector of the Aerodynamic Interface Plane (AIP) (Fig. 4c) and a symmetric pair of counter-rotating vortices (Fig. 4m), often referred to as Dean vortices (Dean 1927). These flow features are the result of the flow separation and secondary flows that arise due to the curvature of the geometry and the diffusion experienced by the flow as previously highlighted by Anderson et al. (1994) and Knoth and Breitsampter (2017). Previous studies highlighted the importance of the dynamic component of the distortion for the stability of the propulsion system, and associated the peak, instantaneous flow distortion events with engine instabilities even when the mean levels were within acceptable limits (Bowditch and Coltrin 1983). The frequency associated with the distorted flow perturbations was also found to have a significant effect on the response of the fan (Wenzel and Blaha 1977). Paul and Younghans (1978) identified a critical frequency that represented an upper limit to the perturbations to which the fan could respond in an unsteady manner. Perturbations associated with higher frequencies are expected to have a negligible effect on the fan, and the engine is then primarily affected by the time-averaged value of these distortions (Breuer and Bissinger 2010). Cousins (2004) defined this critical frequency based on the flow transit time from the leading edge to the throat of the fan rotor. The time in which a rotor blade covers a sector of 4 or 5 blades was proposed as a rule of thumb for the estimation of the upper critical frequency (Cousins 2004).

The highly unsteady nature of the flow within complex intakes (Berens et al. 2014) and the crucial importance of the dynamic distortion on the fan response (Bowditch and Coltrin 1983) have demonstrated the limitations of the conventional measurement methods for inlet flow distortions based on steady pressure probes as well as the standard flow prediction approaches based on steady state RANS models (Fiola and Agarwal, 2015). Delot et al. (2011) measured the unsteady total pressure field at the AIP of a geometry representative of a mild curvature S-duct intake $\left(\mathrm{H} / \mathrm{L}=0.27, \mathrm{AR}=1.52, \mathrm{~L} / \mathrm{D}_{\mathrm{in}}=5.0\right.$, see Fig. 1$)$ at a flow condition of $\mathrm{M}_{\mathrm{AIP}}=0.20\left(\mathrm{Re}_{\mathrm{D}}=7.5 \times 10^{5}\right)$ using 40 high-bandwidth pressure transducers. The spectral analysis revealed that most of the fluctuations were contained within frequencies below $\mathrm{St}=0.75$. Two significant unsteady regions were identified at the lateral edges of the mean loss region (Fig. 4c), and were associated with a main frequency of $\mathrm{St}=0.27$. The pressure signals in these regions were strongly correlated and out of phase, which indicated a lateral oscillation of the flow (Delot et al. 2011). A third region of high unsteadiness was identified at the top edge of the mean loss region, and associated with a main frequency of $\mathrm{St}=0.54$. Garnier (2015) 


\section{Page 5 of $\mathbf{3 6}$}

measured the unsteady total pressure field at the AIP of a more severe $\mathrm{S}$-duct intake $\left(\mathrm{H} / \mathrm{L}=0.49, \mathrm{AR}=1.52, \mathrm{~L} / \mathrm{D}_{\text {in }}=4.95\right)$ at two flow conditions of $M_{\text {AIP }}=0.2\left(\operatorname{Re}_{\mathrm{D}}=7.5 \times 10^{5}\right)$ and $0.4\left(\operatorname{Re}_{\mathrm{D}}=15.0 \times 10^{5}\right)$, also using 40 high-bandwidth pressure transducers. A lateral oscillation was also identified, and associated with a frequency of approximately $\mathrm{St}=0.48$. A region of high unsteadiness at the top edge of the mean loss region was also reported and associated with frequencies between $\mathrm{St}=0.60-1.09$. Based on studies for two intake configurations, Tanguy et al. (2018) used the unsteady distortion descriptor signals to enable the application of the Extreme Value Theory (EVT) to stochastically predict extreme levels of total pressure distortion that could occur based on a reduced, baseline dataset. A similar application of the EVT using unsteady swirl distortion data was presented by Gil-Prieto et al. (2018c) showing that successful application of the theory of extremes can potentially predict peak distortion events beyond the measured ones that could have a detrimental effect on the stability margin of the engine. MacManus et al. (2016) simulated the unsteady total-pressure flow-field within the S-duct geometries studied by Delot et al. (2011) $(\mathrm{H} / \mathrm{L}=0.27)$ and Garnier (2015) $(\mathrm{H} / \mathrm{L}=0.49)$ at similar flow conditions, with a DDES method. For the milder geometry $(\mathrm{H} / \mathrm{L}=0.27)$ at $\mathrm{M}_{\mathrm{AIP}}=0.2$, the lateral and longitudinal perturbations were associated with a main frequency of $\mathrm{St}=0.40$ and 0.75 , respectively. For the more severe configuration $(\mathrm{H} / \mathrm{L}=0.49)$ at $\mathrm{M}_{\mathrm{AIP}}=0.2$, the lateral and vertical oscillation were observed at approximately $\mathrm{St}=0.55$ and 0.85 , respectively. MacManus et al. (2016) also demonstrated that Reynolds and Mach number variations have only a minor effect on the unsteady characteristics of these flow fields.

The work by Zachos et al. (2016) represented a breakthrough in the experimental methods used to quantify distorted flow fields in complex intakes. For the first time, Stereo Particle Image Velocimetry (SPIV) was applied to measure the unsteady, three-component velocity field at the AIP, with approximately 250 times more data points compared with conventional experimental techniques based on 40 pressure transducers. This study considered the same non-dimensional intakes geometries investigated by Delot et al. (2011) and Garnier (2015), at $\mathrm{M}_{\mathrm{ref}}=0.27$ $\left(\operatorname{Re}_{\mathrm{D}}=5.9 \times 10^{5}\right)$ and $0.60\left(\operatorname{Re}_{\mathrm{D}}=13.8 \times 10^{5}\right)$. Zachos et al. (2016) focused the analysis on the unsteady swirl distortion, which can be a notable source of engine instabilities (SAE 2007). For both configurations, the maximum swirl angle fluctuations at the AIP were approximately $\sigma_{\alpha}=15^{\circ}$ and were spread over the lower sector of the section in regions where the time-averaged flow was almost swirl-free. In addition, the unsteady swirl levels were of the same order as the time-averaged values. These results highlighted the need for unsteady swirl measurements such as those obtained with SPIV to account for the highly dynamic nature of the swirl distortion in these intakes (Zachos et al. 2016). GilPrieto et al. (2017a) applied Proper Orthogonal Decomposition (POD) to the measured velocity field at the AIP for 


\section{Page 6 of 36}

the same configurations investigated by Zachos et al. (2016), to identify the fundamental coherent structures in these flow fields. The two most energetic structures for both ducts were a vertical and a switching mode. The vertical mode represented a vertical oscillation of the main loss region (Gil-Prieto et al. 2017a). The switching mode was responsible for the swirl switching mechanism by which alternately one of the Dean vortices becomes dominant in the AIP (Tunstall and Harvey 1968), and leads to bulk swirl patterns that promote the most intense swirl events (Gil-Prieto et al. 2017a). The switching mode was also associated with a lateral movement of the main loss region, which followed the displacement of the dominant vortex. Gil-Prieto et al. (2017b) performed a Delayed Detached Eddy Simulation (DDES) of the flow field within the high offset duct $(\mathrm{H} / \mathrm{L}=0.49)$ studied by Zachos et al. (2016) at $\mathrm{M}_{\text {ref }}=0.27$. The spectral analysis of the POD structures revealed that the switching and vertical modes were associated with dominant frequencies of $\mathrm{St}=0.53$ and 1.06, respectively. Gil-Prieto et al. (2017b) studied the upstream origin of these structures, and showed that the swirl switching was the result of the alternate shedding of clockwise and anti-clockwise streamwise vortices from the separation region. The vertical perturbation was promoted by the shedding of spanwise vortices along the shear layer from the separation region (Gil-Prieto et al. 2017b). It is worth noting that similar switching and vertical structures were also observed in simpler, non-diffusing, single $90^{\circ}$ bends used in piping systems (KalpakliVester et al. 2015; Hellström et al. 2013). Kalpakli-Vester et al.2015 used Time-Resolved SPIV (TR-SPIV) for the measurement of the airflow velocity field downstream of a $90^{\circ}$, non-diffusing bend. In this investigation, the measurements were taken at the exit of the pipe where the flow was discharged into quiescent air. For a bend with a curvature ratio of $\gamma=0.39$ at $\mathrm{Re}_{\mathrm{D}}=2.3 \times 10^{4}$, Kalpakli-Vester et al. (2015) reported a dominant frequency at $\mathrm{St}=0.10$ and a smaller peak at $\mathrm{St}=0.04$ for the switching mode, while the vertical perturbation was associated with a main frequency of $\mathrm{St}=0.04$. Hellström et al. (2013) also used TR-SPIV for the investigation of water flow in a $90^{\circ}$ bend with a curvature ratio of $\gamma=0.50$ at $\operatorname{Re}_{\mathrm{D}}=2.5 \times 10^{4}$. In this case the switching mode was associated with a frequency of $\mathrm{St}=0.33$, while the vertical mode showed a dominant frequency at $\mathrm{St}=0.16$.

The present work builds upon the work of Zachos et al. (2016), and Gil-Prieto et al. (2017a, b), in which SPIV was used to quantify the unsteady, distorted velocity field at the AIP of complex intakes. These previous investigations used a low-bandwidth SPIV system, and even though these measurements allowed the statistical analysis of the distorted flow field, the acquisition frequency was insufficient to resolve the main frequencies in the flow field. The present investigation uses, for the first time, TR-SPIV to quantify the distorted flow field within S-duct intakes. TRSPIV enables the spectral analysis of the distorted flow field, which is of prime importance due to the major effect of 


\section{Page 7 of 36}

the frequency content on the fan response (Breuer and Bissinger 2010). The main aim of this paper is to quantify for the first time the experimental spectral characteristics of the velocity flow field within complex S-duct intakes. The dominant frequencies for two S-duct intakes with different centerline offset at two flow conditions of $\mathrm{M}_{\text {ref }}=0.27$ and 0.60 are identified. The time-resolved velocity data enable spectral analyses for the in-plane and out-of-plane flow features, as opposed to total pressure data where these features are combined and cannot be studied separately. POD is used to elucidate in more detail the spatial and spectral coherent characteristics of the flow. Finally, the spectral analysis of key swirl distortion descriptors correlates their temporal behavior with the identified flow coherent structures.

\section{Experimental Setup and Methods}

\subsection{Experimental facility and test-cases}

An S-duct intake test facility at Cranfield University was used for the experiments. This is described in detail by Zachos et al. (2016). Upstream of the S-duct, the facility includes a bell-mouth intake, a flow conditioning section, a constant diameter section, a conical nozzle that reduces the duct diameter from $200 \mathrm{~mm}$ to $121.6 \mathrm{~mm}$ and finally a straight constant diameter section where a reference measurement plane is located at $1.5 \mathrm{D}_{\text {in }}$ upstream the S-duct inlet (Zachos et al. 2016). This reference plane was used to determine the inlet Mach number to the S-duct based on totalpressure and wall static pressure measurements in order to define the operating point of the rig. The facility was designed in a modular way to accommodate two S-duct configurations (Fig. 1) with different centerline offsets (Table 1). The datum configuration is a scaled version of the geometry investigated by Wellborn et al. (1993) and Delot et al. (2011), and features a centerline offset of $H / L=0.27$, an axial length $L / D_{\text {in }}=5.00$ and an area ratio $A R=1.52$ (Table 1). The higher offset configuration is also a scaled version of the geometry studied by Garnier (2015), with $\mathrm{H} / \mathrm{L}=0.49$, $\mathrm{L} / \mathrm{D}_{\text {in }}=4.95$ and $\mathrm{AR}=1.52$ (Table 1). The AIP diameter for both configurations was $\mathrm{D}_{\mathrm{AIP}}=150 \mathrm{~mm}$. At the exit of the S-duct a transparent section was used to enable optical access for the TR-SPIV laser and cameras. Flow conditions at an inlet reference Mach number of $\mathrm{M}_{\mathrm{ref}}=0.60\left(\mathrm{Re}_{\mathrm{D}}=13.8 \times 10^{5}\right)$ is considered in this work while test were also acquired at a lower reference Mach number of $\mathrm{M}_{\mathrm{ref}}=0.27\left(\mathrm{Re}_{\mathrm{D}}=13.8 \times 10^{5}\right)$. The uncertainty of the reference Mach number was estimated to be approximately $0.6 \pm 0.005$, where the accuracy and resolution of the pressure transducers as well as the 


\section{Page $\mathbf{8}$ of $\mathbf{3 6}$}

calibration uncertainty have been considered and propagated (Taylor 1997). The displacement thickness of the boundary layer at the reference plane was approximately $0.007 \mathrm{D}_{\text {in }}$ at $\mathrm{M}_{\mathrm{ref}}=0.60$.

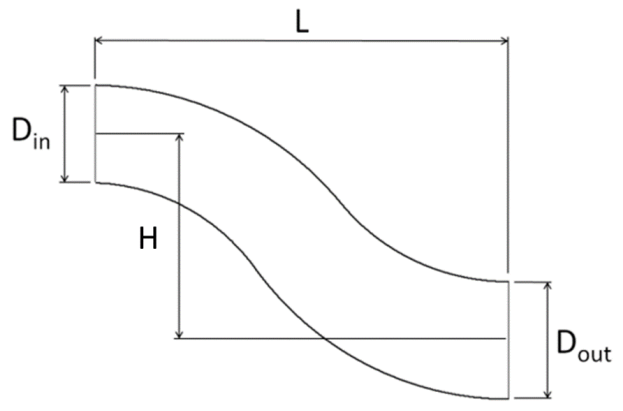

Fig. 1 Schematic definition of S-duct geometry.

Table 1 S-duct geometries

\begin{tabular}{ccc}
\hline \hline Parameter & $\begin{array}{c}\text { High offset } \\
\text { (Duct-1) }\end{array}$ & $\begin{array}{c}\text { Low offset } \\
\text { (Duct-2) }\end{array}$ \\
\hline $\mathrm{A}_{\text {out }} / \mathrm{A}_{\text {in }}$ & 1.52 & 1.52 \\
$\mathrm{~L} / \mathrm{D}_{\text {in }}$ & 4.95 & 5.00 \\
$\mathrm{H} / \mathrm{L}$ & 0.49 & 0.27 \\
\hline
\end{tabular}

\subsection{Time resolved Stereo Particle Image Velocimetry}

Di-Ethyl-Hexyl Sebacate particles were used to seed the flow, and were generated by a Laskin nozzle particle generator at an average diameter of approximately $1 \mu \mathrm{m}$. The seeding particles were illuminated by a dual cavity pulsed Nd:YAG laser at the AIP, located $0.41 \mathrm{D}_{\text {out }}(61 \mathrm{~mm})$ downstream of the exit plane of the intake. A pair of $1 \mathrm{MP}$ CMOS cameras were used in an off-axis stereo arrangement. The cameras featured a sensor with a resolution of 1280x800 px, and a maximum straddle frame rate of 16,600 fps which enables the acquisition of velocity fields with a maximum temporal resolution of $8.3 \mathrm{kHz}$. A pair of AF 1.8/D Nikkor lenses was used for imaging with a focal length of 50mm. A single-plane calibration target was traversed axially to calibrate the cameras. The data acquisition frequency was selected as 10 times the highest dominant flow frequency of $\mathrm{St}=1.06$ as reported previously by GilPrieto et al. (2017b), which resulted in a value of $8 \mathrm{kHz}$ for the two flow condition of $\mathrm{M}_{\mathrm{ref}}=0.60$. Each dataset comprised 20,000 instantaneous velocity fields, which was sufficient to achieve statistically converged results. For 


\section{Page 9 of 36}

example, $\langle\bar{w}\rangle_{A I P}$ and $\bar{\sigma}_{w_{A I P}}$ converged to within $0.2 \%$ and $0.4 \%$ after 15000 snapshots, respectively, and similar results were obtained for the other velocity components.

The PIV processing algorithm used a direct correlation method, and a recursive multi-pass window approach was employed with an initial window size of $128 \times 128$ px, and a final window size of 32x32 px, with a 50\% overlap. The PIV processing strategy resulted in a vector resolution of approximately $0.0187 \mathrm{D}_{\text {out }}(2.8 \mathrm{~mm})$ in both directions across the AIP. Only data within $r<0.9 R_{A I P}$ is reported in the present work to remove the effect of spurious vectors near the domain's boundaries due to laser light reflections. The total number of velocity vectors acquired across the AIP within $r<0.9 R_{A I P}$ is approximately 1800 . Bias errors due to misalignment between the light sheet and the calibration plate were mitigated with an iterative disparity correction embedded onto LaVision's DaVis 8.3 software and previously reported by Wieneke (2005). The uncertainty on the velocity components has been estimated by means of the method described by Raffel et al. (2007), which accounts for the effect of the particle image displacement, particle image diameter, particle density in the interrogation window, image quantization levels, particle displacement gradient and background noise. The uncertainty of the calibration polynomials were considered, and the estimated uncertainties for the in-plane and out-of-plane velocities are respectively $4.9 \%$ and $7.0 \%$ of the area-averaged, time-averaged $w$ velocity at the AIP, $\langle\bar{w}\rangle_{A I P}$.

\subsection{Proper Orthogonal Decomposition}

Proper Orthogonal Decomposition (POD) (Berkooz et al. 1993) enables the identification of the flowfield coherent structures, and has been previously applied across a wide range of aerodynamic applications including backwardfacing steps (Kostas et al. 2002, 2005), curved pipes (Kalpakli-Vester et al. 2015; Hellström et al. 2013) and more recently S-ducts (Gissen et al. 2014; MacManus et al. 2016; Gil-Prieto et al. 2017a, b). The POD of the unsteady velocity vector field $\boldsymbol{V}^{\prime}=\boldsymbol{V}-\langle\boldsymbol{V}\rangle$ identifies an orthonormal set of bases $\boldsymbol{\Phi}_{\boldsymbol{i}}=\left\{\Phi_{i}^{u}, \Phi_{i}^{v}, \Phi_{i}^{w}\right\}$, which are referred to as POD modes and are invariant with time. Each POD mode is associated with a temporal coefficient $a_{i}(t)$, and the contribution to the original unsteady velocity field from each POD structure is $a_{i}(t) \boldsymbol{\Phi}_{\boldsymbol{i}}$. Therefore, the POD enables a low dimensional representation of the original flow field using the first $k$-modal contributions $\boldsymbol{V}_{1-k}$ (Eq. 1) (Berkooz et al. 1993). The variance of each temporal coefficient $\left\langle a_{j}{ }^{2}\right\rangle$ represents the contribution of each POD structure to the area-averaged mean TKE (Eq. 2), and the POD modes are ordered by its associated TKE contribution. The POD low- 


\section{Page 10 of 36}

dimensional representation (Eq. 1) is then optimal since it maximizes the TKE in the reconstructed flowfield for a given number of $k$ terms in the series (Lumley 2007).

$$
\begin{gathered}
\boldsymbol{V}_{1-k}=\langle\boldsymbol{V}\rangle+\sum_{i=1}^{k} a_{i}(t) \boldsymbol{\Phi}_{i}(x, y) \\
\langle\overline{T K E} \bar{E}\rangle_{1-k}=\sum_{i=1}^{k}\left\langle a_{i}{ }^{2}\right\rangle
\end{gathered}
$$

\subsection{Swirl distortion descriptors}

In order to characterize and quantify the distortion patterns presented to the engine front face a number of swirl distortion descriptors was previously developed (SAE, 2007). In this definition, the AIP is divided into several radial rings and the swirl descriptors are evaluated at each radial position based on the ring swirl angle distribution for each flow snapshot. A sample twice-per-revolution swirl pattern is shown in Fig. 2 illustrating the definitions of the swirl distortion descriptors. The swirl angle is positive in the counterclockwise direction when the AIP is considered from downstream. Positive and negative circumferential extents, $\theta_{i, k}^{+}$and $\theta_{i, k}^{-}$define the size of the k-th swirling region in the $i$-th ring. Positive and negative sector swirls $S S_{i, k}^{+}$and $S S_{i, k}^{-}$are defined as the mean swirl-angle value in each of the $\mathrm{m}$ swirling regions (SAE, 2007).

Sector swirl and extents are used as the foundation for the development of a range of swirl descriptors used to quantify the nature and strength of the swirl distortion. Swirl intensity (SI) reflects the averaged absolute swirl angle in each radial ring (Eq. 3). Swirl directivity (SD) shows the overall sense of rotation of the swirling flow (Eq. 4) and Swirl Pairs (SP) indicates the number of swirl pairs relative to the swirl region in the ring which encloses the highest absolute swirl angle content, $\max \left(S S_{i, k}^{+} \theta_{i, k}^{+},\left|S S_{i, k}^{-} \theta_{i, k}^{-}\right|\right)$(Eq. 5). The descriptors are typically evaluated at five equal area rings that makes it consistent with common industrial practice based on conventional pressure probe that are used to measure the distortion patterns (SAE, 2007). At each ring, 72 equi-spaced circumferential points are considered, which results in a resolution of $5 \mathrm{deg}$. The S-PIV data is linearly interpolated at those locations using the Delaunay triangulation method (Lee et al. 1980). 


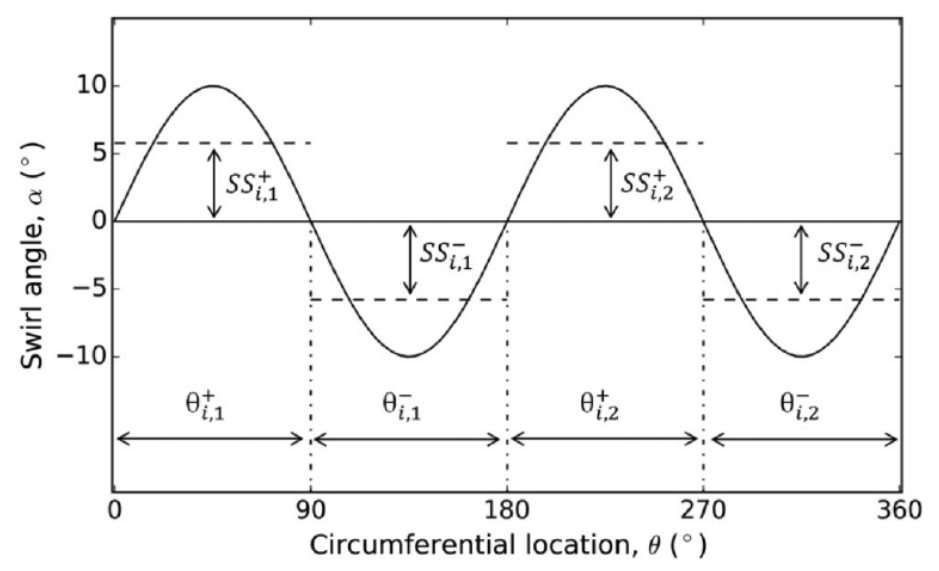

Fig. 2 Twice-per-revolution swirl distortion pattern (SAE, 2007).

$$
\begin{gathered}
S I(i)=\frac{\sum_{k=1}^{m} S S_{i, k}^{+} \cdot \theta_{i, k}^{+}+\sum_{k=1}^{m}\left|S S_{i, k}^{-}\right| \cdot \theta_{i, k}^{-}}{360} \\
S D(i)=\frac{\sum_{k=1}^{m} S S_{i, k}^{+} \cdot \theta_{i, k}^{+}+\sum_{k=1}^{m} S S_{i, k}^{-} \cdot \theta_{i, k}^{-}}{\sum_{k=1}^{m} S S_{i, k}^{+} \cdot \theta_{i, k}^{+}+\sum_{k=1}^{m}\left|S S_{i, k}^{-}\right| \cdot \theta_{i, k}^{-}} \\
S P(i)=\frac{\sum_{k=1}^{m} S S_{i, k}^{+} \cdot \theta_{i, k}^{+}+\sum_{k=1}^{m}\left|S S_{i, k}^{-}\right| \cdot \theta_{i, k}^{-}}{2 \cdot \operatorname{Max}\left\{S S_{i, k}^{+} \cdot \theta_{i, k}^{+},\left|S S_{i, k}^{-} \cdot \theta_{i, k}^{-}\right|\right\}_{k=1, \ldots, m}}
\end{gathered}
$$

A possible way to characterize, for example, the unsteady swirl distortion patterns, both SP and SD can be considered as a part of a common cloud map as previously shown by Gil-Prieto et al. (2017b) and Zachos et al. (2016). These maps indicate the range and trend between the descriptors, and can be used as an illustration of the AIP distorted flow topologies at instantaneous snapshot that can oscillate across a range between negative or positive bulk swirl (Fig. 3). If the relative frequency of occurrence of the events in different regions of the map is included, the characterization of the distortion patterns would include the distinction between the rare and common events. For this work, the distortion maps include this probabilistic aspect whereby the joint Probability Density Function (join-PDF) for the two descriptors is represented. For the evaluation of the joint-PDF, the range of each descriptor has been discretized in 60 equi-spaced partitions. This results in a resolution of approximately 0.03 for SP and SD. The probability to identify the distorted pattern within a given region of this SP-SD map is obtained by integration of the PDF over the desired area (Eq. (6)).

$$
P\left(S D_{A} \leq S D<S D_{B}, S P_{A} \leq S P<S P_{B}\right)=\int_{S P_{A}}^{S P_{B}} \int_{S D_{A}}^{S D_{B}} P D F d S D d S P
$$




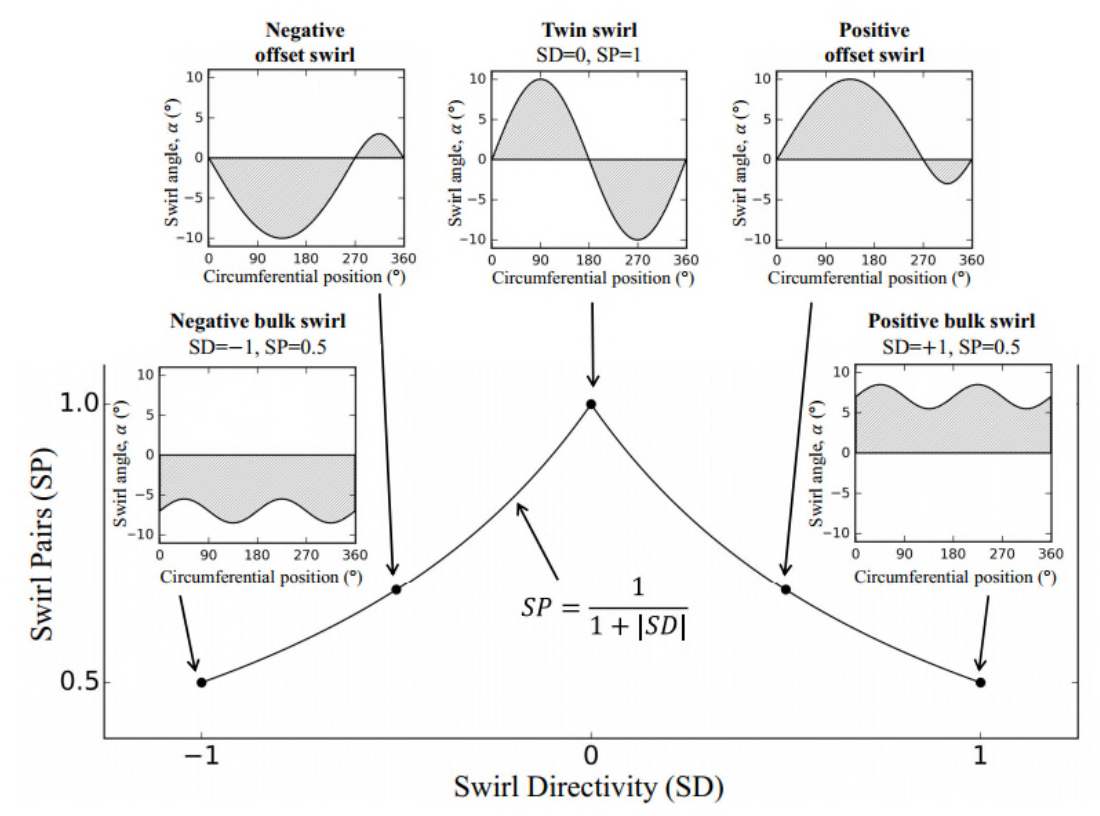

Fig. 3 One-per-revolution bulk-to-twin swirl switching path.

\section{Results and Discussion}

\subsection{Mean and unsteady flow field analysis}

In this section the time-averaged and standard-deviation distributions of the velocity components and swirl angle at the AIP for the high and low offset configurations at $\mathrm{M}_{\mathrm{ref}}=0.60$ are presented (Fig. 4). In all figures hereafter, $u$ is used to indicate the horizontal in-plane velocity along the $\mathrm{x}$-axis, $v$ for the vertical in-plane velocity along the $\mathrm{y}$-axis and $w$ for the out of plane velocity component along the z-axis. A detailed discussion was provided by Zachos et al. (2016) based on low-bandwidth SPIV data, and only the key aspects are discussed here. For Duct-1 (H/L=0.49) the time-averaged $w$-velocity shows the typical loss region at the lower sector of the AIP (Fig. 4a), and the maximum $w$ velocity fluctuations of about $\operatorname{std}(w) /\langle\bar{w}\rangle_{A I P}=0.17$ occur at the upper edge of this loss region (Fig. 4b). A small region of high $w$-velocity fluctuations is located near the top wall, with values of approximately $\operatorname{std}(w) /\langle\bar{w}\rangle_{A I P}=0.13$ (Fig. 4b). The time-averaged distributions of $u$ (Fig. 4e) and $v$-velocity (Fig. 4i) result in the expected pair of vortices located at the bottom part of the AIP (Fig. 4m). Previous work showed these distributions to be symmetric with regards to the y-axis of the AIP (Gil et al., 2017a, Gil et al. 2017b). This symmetry is also broadly shown in most of the time- 


\section{Page 13 of 36}

averaged and unsteady distributions at the AIP (Fig. 4, Fig. 5, Fig. 6). However, there are minor asymmetries present in some of the AIP profiles as for example in Fig. 4c, Fig. 4d and Fig. 5b. Nevertheless, the main characteristics of the flow are well represented and are generally in good agreement with previous computational research (Gil et al., 2017a, Gil et al. 2017b). The maximum $u$-velocity fluctuations occur in the lower sector of the AIP (Fig. 4f) with similar values to those of the $w$-velocity (Fig. 4b). The fluctuations of the $v$-velocity fluctuations are spread over most of the AIP, and the maximum values of $s t d(v) /\langle\bar{w}\rangle_{A I P}=0.22($ Fig. $4 \mathrm{j})$ are greater compared with the other velocity components (Fig. 4b,f). The maximum swirl angle fluctuations of $\operatorname{std}(\alpha)=14.1^{\circ}$ occur at a centered region of the AIP (Fig. $4 \mathrm{n}$ ), and are greater than the maximum local time-averaged values of $\langle\alpha\rangle=9.6^{\circ}$ (Fig. $4 \mathrm{~m}$ ). The low offset Duct-2 $(\mathrm{H} / \mathrm{L}=0.27)$ also features the typical mean loss region (Fig. 4c) and the symmetric pair of vortices (Fig. 4o). However, compared with the high offset duct, in Duct-2 $(\mathrm{H} / \mathrm{L}=0.27)$ the loss region covers a smaller area located closer to the lower wall (Fig. 4c), and the vortices are weaker and promote lower swirl levels (Fig. 4o). In the low offset Duct-2, the maximum $w$-velocity fluctuations also occur at the upper boundary of the main loss region (Fig. 4d), with maximum values $\operatorname{std}(w) /\langle\bar{w}\rangle_{A I P}=0.19$ slightly greater than those in Duct-1 (Fig. $4 \mathrm{~b}$ ). The fluctuations of swirl angle in Duct-2 $(\mathrm{H} / \mathrm{L}=0.27)$ are located near the lower wall (Fig. $4 \mathrm{p})$, as opposed to the more central position observed in the high offset Duct-1 (Fig. 4n). The Mach number has only a minor effect on the flowfield characteristics (Zachos et al. 2016), and similar results are obtained at $\mathrm{M}_{\mathrm{ref}}=0.27$ for both configurations. Overall, these results highlight the unsteady nature of these flow fields, and the need for SPIV that can provide synchronous measurements across the AIP with high spatial-resolution. 
High-offset $(H / L=0.49), M_{\text {ref }}=0.60$

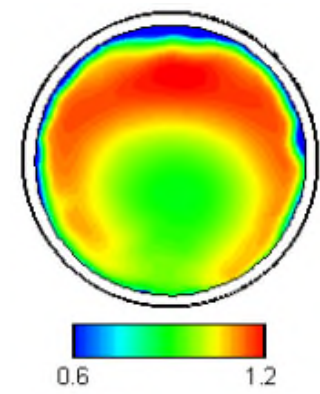

(a) $\langle w\rangle /\langle\bar{w}\rangle_{A I P}$

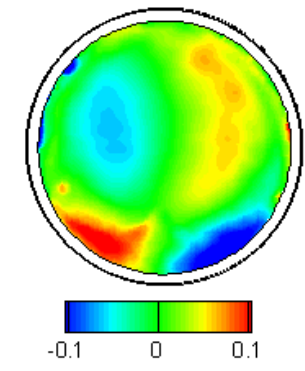

(e) $\langle u\rangle /\langle\bar{w}\rangle_{A I P}$

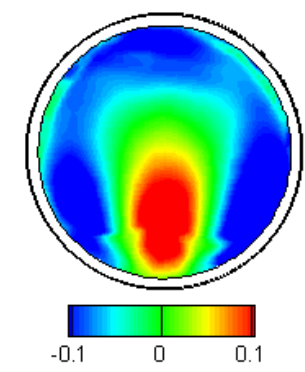

(i) $\langle v\rangle /\langle\bar{w}\rangle_{A I P}$

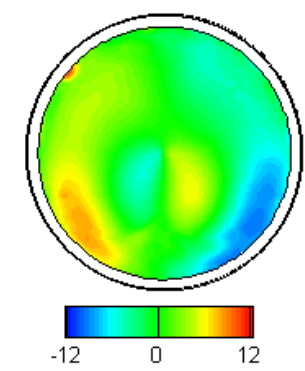

(m) $\langle\alpha\rangle(\operatorname{deg})$

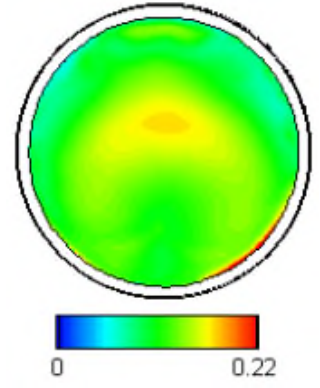

(b) $\operatorname{std}(w) /\langle\bar{w}\rangle_{A I P}$

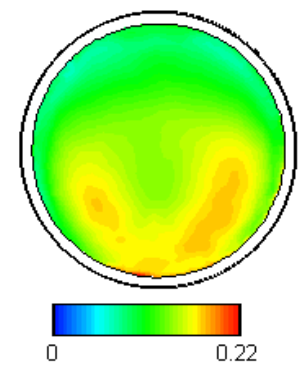

(f) $\operatorname{std}(u) /\langle\bar{w}\rangle_{A I P}$

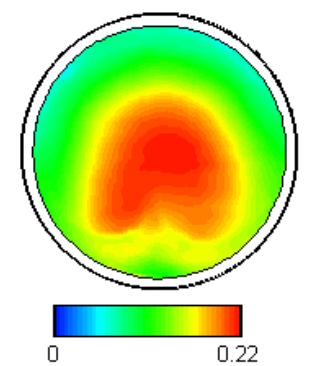

(j) $\operatorname{std}(v) /\langle\bar{w}\rangle_{A I P}$

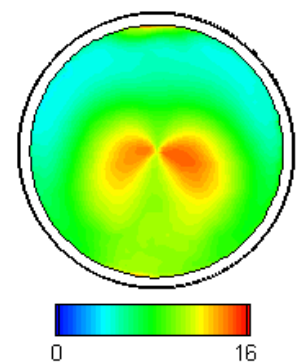

(n) $\operatorname{std}(\alpha)(\mathrm{deg})$
Low-offset $(H / L=0.27), M_{\text {ref }}=0.60$

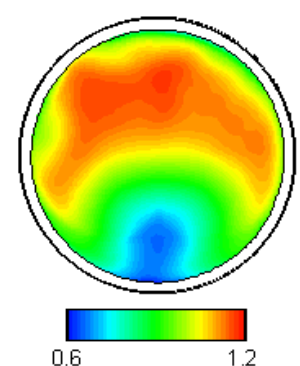

(c) $\langle w\rangle /\langle\bar{w}\rangle_{A I P}$

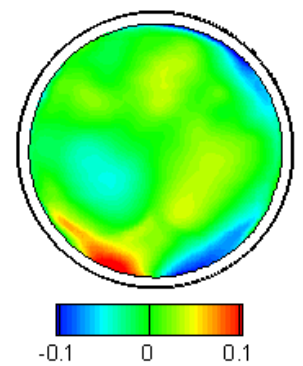

(g) $\langle u\rangle /\langle\bar{w}\rangle_{A I P}$

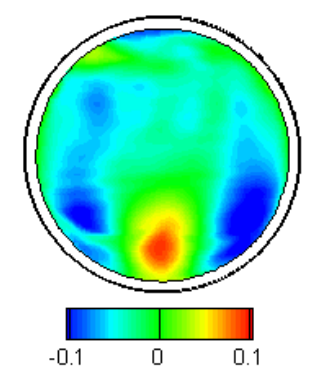

(k) $\langle v\rangle /\langle\bar{w}\rangle_{A I P}$

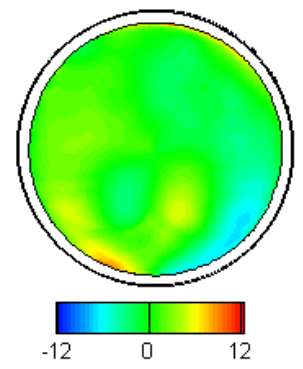

(o) $\langle\alpha\rangle(\mathrm{deg})$

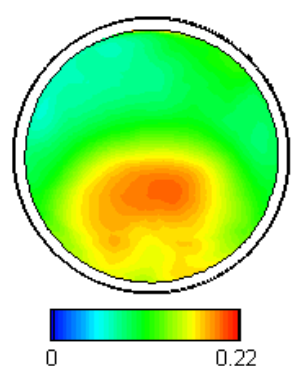

(d) $\operatorname{std}(w) /\langle\bar{w}\rangle_{A I P}$

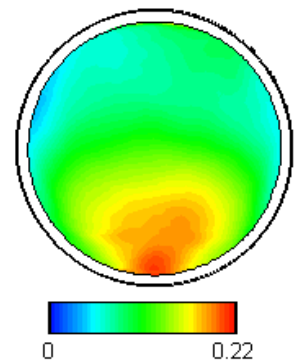

(h) $\operatorname{std}(u) /\langle\bar{w}\rangle_{A I P}$

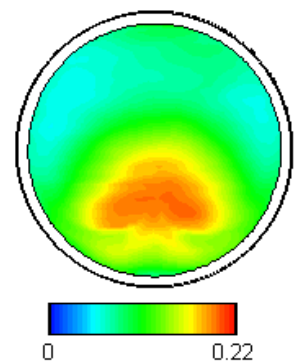

(l) $\operatorname{std}(v) /\langle\bar{w}\rangle_{A I P}$

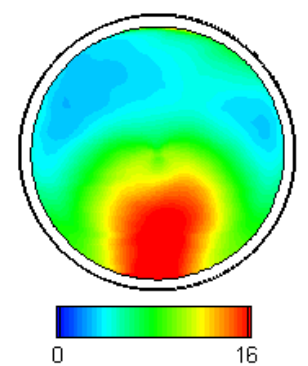

(p) $\operatorname{std}(\alpha)(\operatorname{deg})$

Fig. 4 AIP flowfield statistics for Duct-1 $(\mathrm{H} / \mathrm{L}=0.49)$ and Duct-2 $(\mathrm{H} / \mathrm{L}=0.27)$ at $\mathrm{M}_{\mathrm{ref}}=\mathbf{0 . 6 0}$. 


\section{Page 15 of 36}

\subsection{Velocity field spectral analysis}

The frequencies of these unsteady distorted flow fields affect the response of the fan and significantly impact the loss of surge margin for a given distortion intensity (Wenzel et al. 1977). Therefore, the study of their spectral characteristics is of great importance to anticipate potential incompatibilities between the intake and the engine, and has been previously assessed with high-bandwidth total pressure measurements (Delot et al. 2011; Garnier 2015). However, these previous studies did not allow the distinction between in-plane and out-of-plane flow mechanisms, and provided a limited spatial resolution. In this work, the first spectral analysis of the three components of the velocity vector field at the AIP based on experimental data of high-spatial resolution is presented. The maximum critical frequency to which the fan is likely to respond is estimated to approximately be within the range $\mathrm{St}=0.9-5.4$ ( $\mathrm{Strazisar}$ et al. 1989). The lower limit is estimated with the traditional assumption that critical frequency is approximately the first engine order frequency (Paul and Younghans 198), while the upper limit is estimated based on the time that takes for the rotor blades to travel over a typical critical sector of 60 deg (Reid 1969).

The power spectrum (PS) of the three velocity components has been evaluated at each point of the AIP using an average periodogram method (Welch 1967) for both S-duct configurations at $\mathrm{M}_{\text {ref }}=0.60$ and 0.27 . The complete datasets of 20,000 snapshots have been split in 20 segments of 1000 snapshots, and the overall power spectrum has been evaluated as the ensemble average of the PS of these segments. A Hanning window was applied in each of the segments to avoid frequency leakage, and the time-averaged value of the local velocity at each point has been subtracted prior to the PS computation. The maximum frequency which can be identified in the spectrum is approximately $\mathrm{St}=5.0$, and the frequency resolution is approximately $\Delta \mathrm{St}=0.01$. To enable the visualization of the frequency content, the contribution to the overall variance from different frequency bands has been calculated across the measurement plane for the low offset S-duct configuration (Fig. 5). The contribution of each frequency band to the overall unsteadiness can be quantified as the proportion of the area-averaged value of the variance accounted for by each frequency band (Fig. 5 and Fig. 6). For example, the contribution of the frequency band [St $\mathrm{St}_{1} \mathrm{St}_{2}$ ) to the overall $T K E$ is expressed as $\overline{\operatorname{Var}(w)}_{S t \in\left[S t_{1}, S t_{2}\right)} /\langle\overline{T K E}\rangle$.

For the low offset configuration with $\mathrm{H} / \mathrm{L}=0.27$ at $\mathrm{M}_{\mathrm{ref}}=0.60$, the maximum $w$-velocity fluctuations $\left(\overline{\operatorname{Var}(w)}_{S t \in[0.2,0.4)} /\langle\overline{T K E}\rangle=8 \%\right)$ are associated with frequencies St between 0.2-0.4 (Fig. 7a). The $w$-velocity variance contribution from this frequency band shows an almost symmetric arrangement, with one region of high unsteadiness at each side of the vertical symmetry axis, in the lower sector of the AIP (Fig. 5b) around the lateral 


\section{Page 16 of 36}

edges of the mean loss region (Fig. 4c). At higher frequencies $(\mathrm{St}=0.4-1.0)$, the $w$-velocity fluctuations are located at a more central region of the AIP, at the top edge of the mean loss region (Fig. 5c,d,e). Note that an important contribution to the $w$-velocity fluctuations $\left.(\overline{\operatorname{Var}(w)})_{S t \in[0,0.2)} /\langle\overline{T K E}\rangle=4 \%\right)$ also comes from the lowest frequency band between $\mathrm{St}=0.0$ and 0.2 (Fig. $7 \mathrm{a}$ ). In this band, the unsteadiness is mainly located at the lower sector of the AIP (Fig. 5a). The contribution to the $w$-velocity unsteadiness reduces monotonically at frequencies above the dominant $\mathrm{St}=0.2-0.4$ band, and approximately $35 \%$ of the $w$-velocity fluctuations are contained within $S t<1.2$ (Fig. $7 \mathrm{a}$ ). The contribution from the different frequency bands to the overall $w$-velocity unsteadiness is also almost identical across the range of Mach numbers studied between $\mathrm{M}_{\mathrm{ref}}=0.27$ and $\mathrm{M}_{\mathrm{ref}}=0.60$ which indicates that the inlet Mach number has only a secondary effect on the flow unsteady characteristics.

The high offset $\mathrm{S}$-duct configuration $(\mathrm{H} / \mathrm{L}=0.49)$ at $\mathrm{M}_{\mathrm{ref}}=0.60$ shows the same qualitative unsteady flow features as the low offset $\mathrm{S}-$ duct $(\mathrm{H} / \mathrm{L}=0.27)$, even though there is a notable change in the frequencies associated with the different flow mechanisms (Fig. 6). A pair of regions of high $w$-velocity fluctuations that are broadly symmetric relative to the vertical symmetry axis is found within the band $\mathrm{St}=0.4-0.6$ (Fig. $6 \mathrm{c}$ ), and are located around the mean shear layer (Fig. 4a). The associated perturbations of the in-plane velocity components within $\mathrm{St}=0.4-0.6$ show a region of high $u$-velocity fluctuations near the lower wall (Fig. 6i) and two symmetric regions of high $v$-velocity fluctuations (Fig. 6o). The distribution of the velocity components fluctuations within $\mathrm{St}=0.4-0.6$ in Duct-1 (H/L=0.49) (Fig. 6c,i,o) resembles that for the low-offset configuration $(\mathrm{H} / \mathrm{L}=0.27)$ within $\mathrm{St}=0.2-0.4$ (Fig. 5b,h,n). The main difference was found in the location of the unsteady regions of the $w$-velocity, due to the different position of the mean shear layer which is closer to the lower wall in the low offset configuration (Fig. 4a,c). A region of high w- (Fig. 6d,e) and vvelocity (Fig. 6p,q) fluctuations located at the top edge of the mean loss region is also found for the high offset configuration at higher frequencies $\mathrm{St}=0.6-1.0$. These characteristics are similar to those observed in the low offset Duct-2 within $\mathrm{St}=0.4-1.0$ (Fig. 5c,d,e,o,p,q). However, in Duct-2 (H/L=0.27) these unsteady regions are closer to the lower wall due to the relocation of the mean shear layer (Fig. 4a,c). As opposed to the low offset Duct-2, Duct-1 $(\mathrm{H} / \mathrm{L}=0.49)$ shows a small region of high $w$-velocity fluctuations near the top wall which shows a broadband spectrum (Fig. 6a,b,c,d,e). As for the low offset case, in the high offset duct the Mach number $\mathrm{M}_{\mathrm{ref}}$ does not significantly alter the unsteadiness distribution across the different frequency bands, and similar results are obtained at $\mathrm{M}_{\mathrm{ref}}=0.60$ (Fig. 6) and $\mathrm{M}_{\mathrm{ref}}=0.27$. 


\section{Page 17 of 36}

Overall, the main frequencies in these flows are within the range $\mathrm{St}<1.2$, and are therefore within the estimated critical frequency range $\left(\mathrm{St}_{\text {crit }}=0.9-5.4\right)$ to which a fan rotor is likely respond. Therefore, the introduced unsteadiness could affect the operability of a typical aero-engine. 


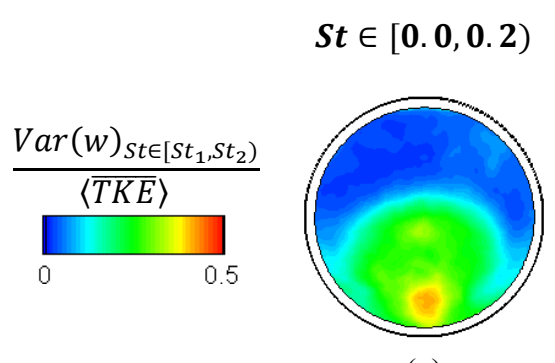

(a)
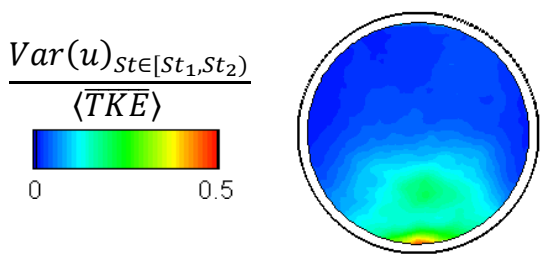

(g)

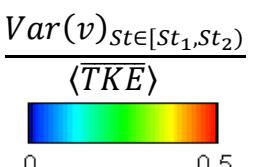

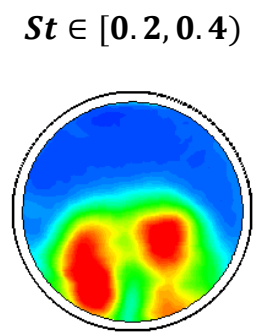

(b)
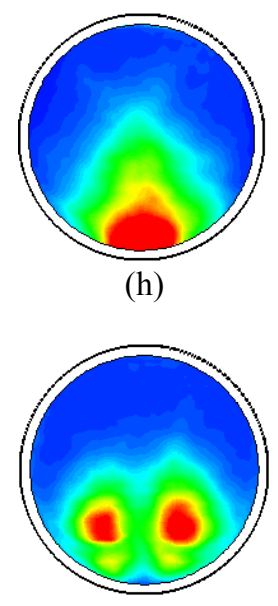

(n)

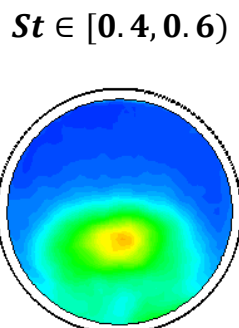

(c)

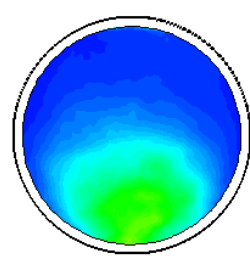

(i)

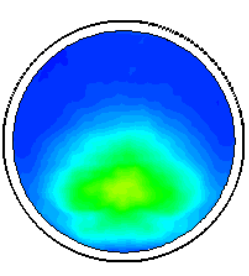

(o)

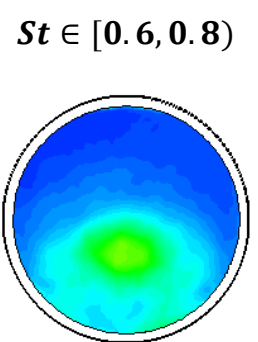

(d)
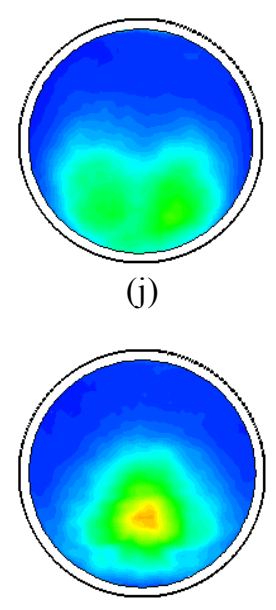

(p)
$S t \in[0.8,1.0)$

$S t \in[1.0,1.2)$

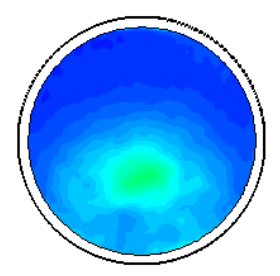

(e)
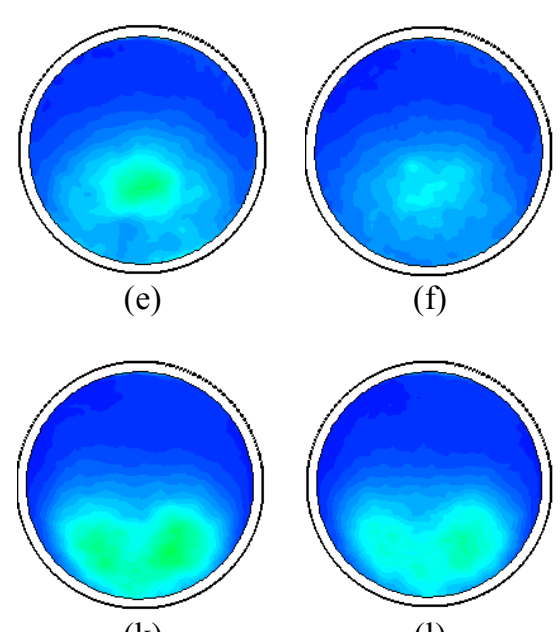

(k)
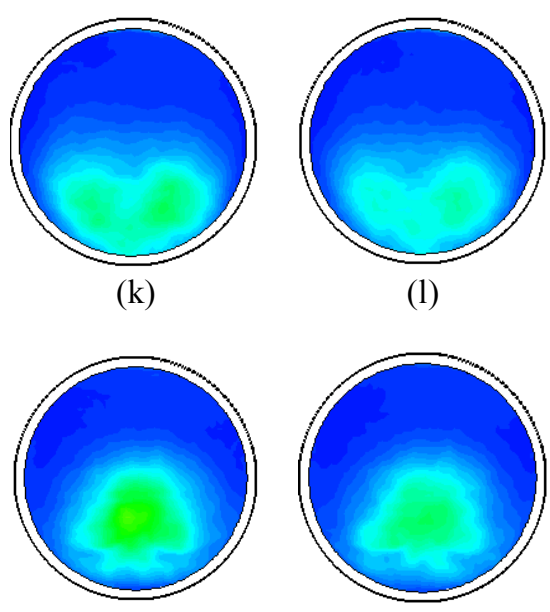

(q)

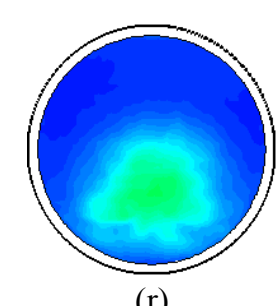

Fig. 5 Spectral distribution of the velocity fluctuations at the AIP for the low-offset S-duct $(H / L=0.27)$ at $M_{\text {ref }}=0.60$. 


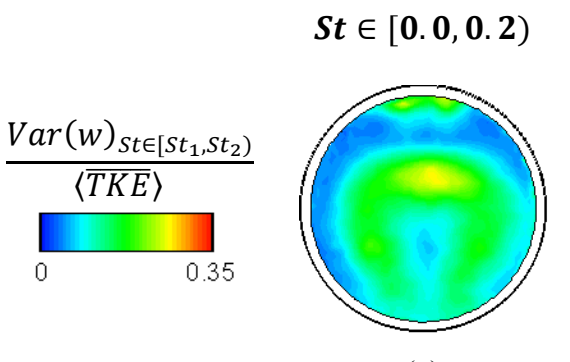

(a)
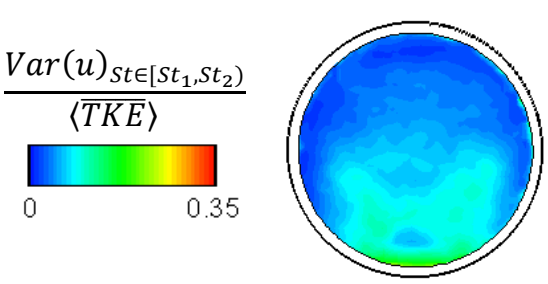

(g)

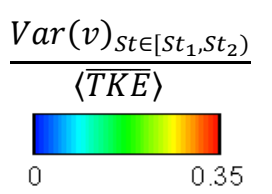

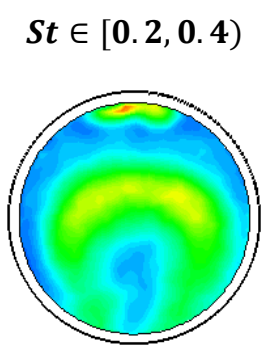

(b)

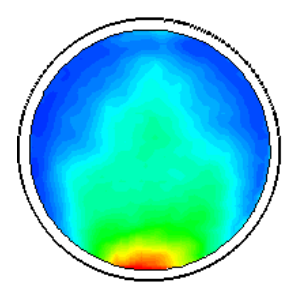

(h)

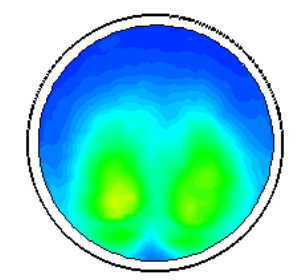

(n)

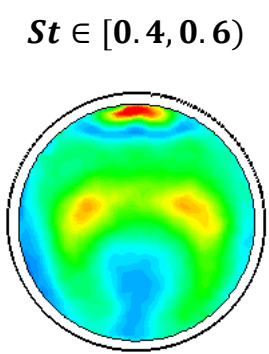

(c)

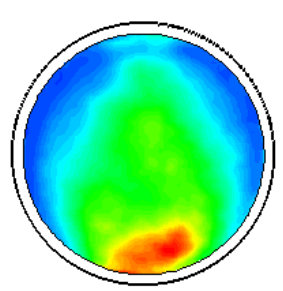

(i)

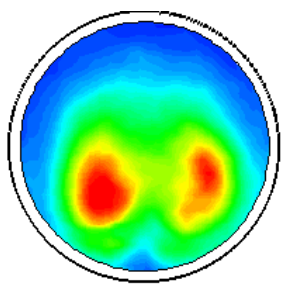

(o)

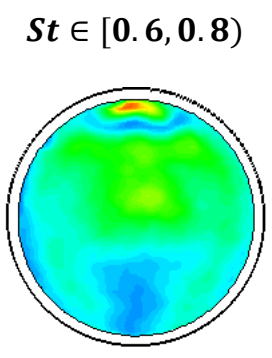

(d)

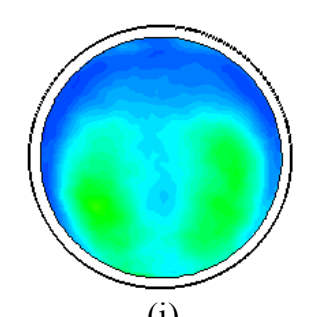

(j)

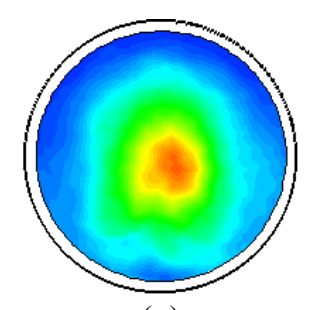

(p)

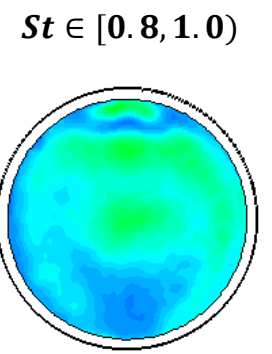

(e)

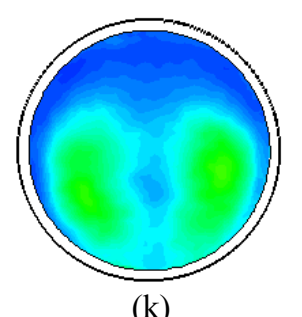

(k)

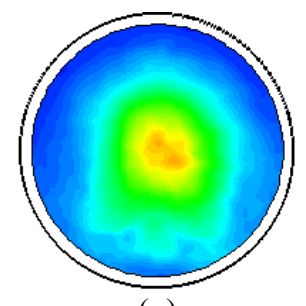

(q)
$S t \in[1.0,1.2)$
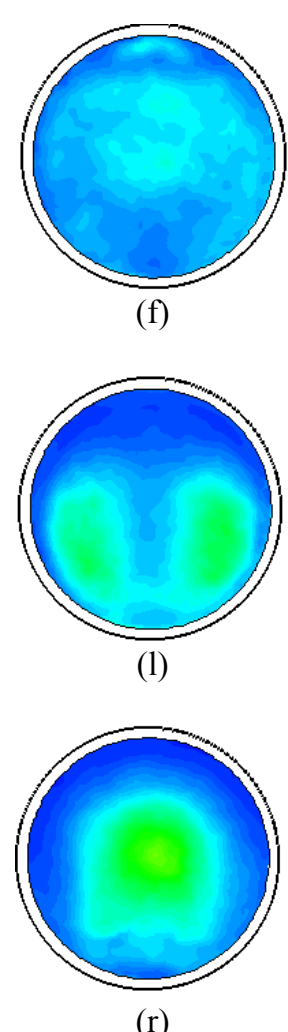

Fig. 6 Spectral distribution of the velocity fluctuations at the AIP for the high-offset S-duct $(H / L=0.49)$ at $M_{\text {ref }}=0.60$. 
Page 20 of 36

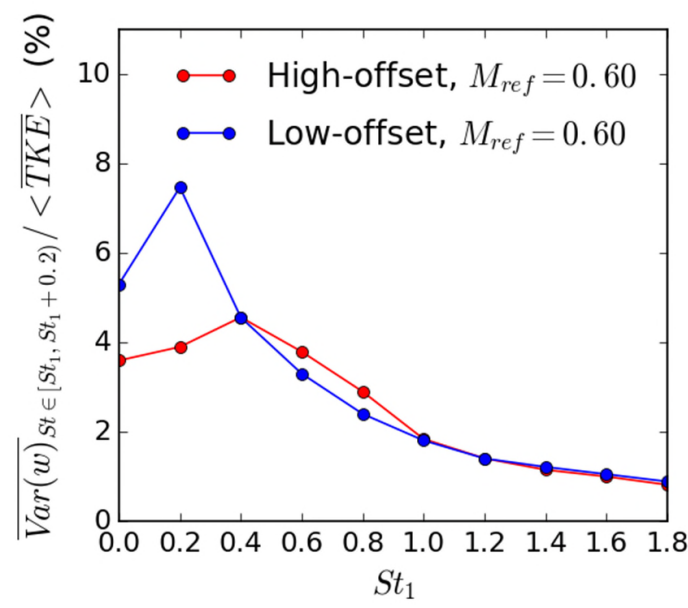

(a)

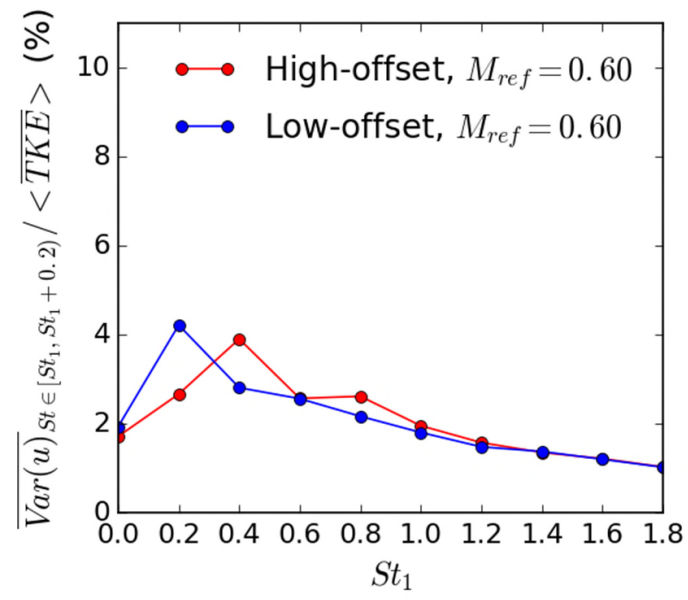

(b)

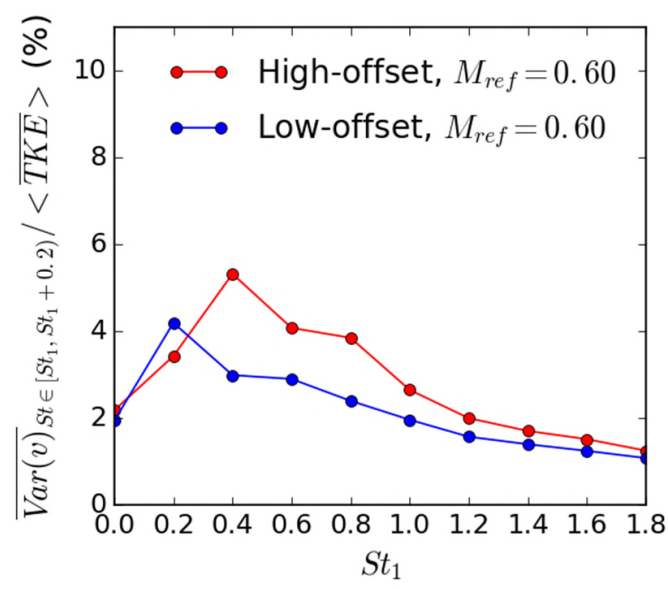

(c)

Fig. 7 Spectral distribution of the variance of the AIP velocity components. 
Page 21 of 36

\subsection{Coherent structure identification and spectral analysis via POD}

The spectral analysis developed in Section 3.2 indicated the presence of two main structures in the flow, which are qualitatively similar in both ducts, even though there are differences in terms of their exact topology and frequency content. Gil-Prieto et al. (2017a) previously applied POD to the measured AIP velocity field within the high offset configuration $(\mathrm{H} / \mathrm{L}=0.49)$ at $\mathrm{M}_{\mathrm{ref}}=0.27$ using low-bandwidth SPIV data, and showed that the flow is dominated by two main flow mechanisms: the swirl switching and the shear-layer unsteadiness. The swirl switching promoted the alternate dominance of one of the two Dean-vortices observed in the mean flow (Fig. 4m), with a concomitant lateral oscillation of the main loss region (Gil-Prieto et al. 2016a). The shear-layer unsteadiness mainly represented a fluctuation of the $v$-velocity field associated with an out-of-phase $w$-velocity fluctuation at the top edge of the mean loss region. Gil-Prieto et al. (2017b) showed that the swirl switching was described by two POD modes, the First Switching Mode (FSM) and Second Switching Mode (SSM), while the shear layer unsteadiness was mainly promoted by the First Vertical Mode (FVM) and Second Vertical Mode (SVM). However, the frequency spectral characteristics of these flow structures has not been previously determined experimentally. In this section, Proper Orthogonal Decomposition (POD) is applied to the three-component velocity vector measured at the AIP to experimentally characterize the frequency spectrum of these flow mechanisms for the first time. Such an analysis is arguably of critical importance for the identification of the stability of the propulsion system due to the susceptibility of the fan response to the frequency content of the distorted flow field (Breuer and Bissinger 2010).

In the present work, the four most energetic POD modes in the high offset Duct- 1 at $\mathrm{M}_{\mathrm{ref}}=0.60$ show the same qualitative spatial distributions as the FVM (Fig. 8a,b), FSM (Fig. 8c,d), SVM (Fig. 8e,f) and SSM (Fig. 8g,h) identified by Gil-Prieto et al. (2017b), and are named accordingly. The FVM, FSM, SVM, and SSM account for approximately $16.4 \%, 14.5 \%, 6.3 \%$ and $3.9 \%$ of the overall $\overline{T K E}$, respectively, and together represent $40 \%$ of the overall $\overline{T K E}$ of the original flow field. The FVM mainly represents $w$-velocity fluctuations at the center of the AIP (Fig. 8a) associated with out-of-phase in-plane velocity unsteadiness (Fig. 8b). The PS of the FVM temporal coefficient $a_{F V M}$ (Fig. 9a) shows significant contributions mainly between $\mathrm{St}=0.6$ and 1.0. Therefore the FVM is mainly responsible for the out-of-phase $w$ - and in-plane velocity fluctuations across a range between $\mathrm{St}=0.6-1.0$ at the top edge of the main loss region. The first switching mode (FSM) represents a single rotating structure (Fig. 8c) that changes alternatively its direction of rotation, at a frequency of approximately $\mathrm{St}=0.42$ (Fig. 9b). This in-plane perturbation is associated with an out-of-phase oscillation of the $w$-velocity at each side of the vertical symmetry axis 


\section{Page 22 of 36}

(Fig. 8c), which describes a lateral oscillation of the main loss region (Gil-Prieto et al. 2017b). As demonstrated by Gil-Prieto et al.(2016b) the FVM (Fig. 8a,b) and SVM (Fig. 8e,f) describe the effect on the AIP of a street of spanwise vortices shed from the upstream separation region. Similarly, the FSM (Fig. 8c,d) and SSM (Fig. 8g,h) together describe the perturbations on the AIP created by the alternate shedding of counter-rotating streamwise vortices from the separation region. As previously indicated by Gil-Prieto et al. (2017b) using numerical results, the SVM (Fig. 9c) shows very similar frequency content as the FVM (Fig. 9a). The same observation stands for the frequency content between the SSM and FSM (Fig. 9d and Fig. 9b). The frequency content obtained for the FSM (Fig. 9b) and SSM (Fig. 9d) with a single dominant peak at $\mathrm{St}=0.45$ is also in agreement with the DDES performed by Gil-Prieto et al. (2017b), which predicted a dominant peak at $\mathrm{St}=0.53$. The spectrum of the FVM (Fig. 9a) and SVM (Fig. 9c) show a range of dominant frequencies between $\mathrm{St}=0.6$ and roughly 1.0 , which is also in broad agreement with the dominant range between $\mathrm{St}=0.72$ and 1.06 reported by Gil-Prieto et al.(2017b). However, in the present work the PS of FVM (Fig. 9a) and SVM (Fig. 9c) show a more broadband spectrum, while the computational results reported by Gil-Prieto et al. (2017b) showed a much more distinct peak at $\mathrm{St}=1.06$. The Mach number $\mathrm{M}_{\mathrm{ref}}$ has only a minor effect on the spatial distribution of the POD modal structures and the energy content, which at $\mathrm{M}_{\mathrm{ref}}=0.27$ is $17.2 \%, 12.1 \%, 6.0 \%$ and 3.8\% for the FVM, FSM, SVM and SSM. The temporal coefficient PS was also found to be similar at $\mathrm{M}_{\mathrm{ref}}=0.27$.

Qualitatively similar structures are obtained for the low offset S-duct configuration (Fig. 10), with small changes in the topology and position of the structures due to the relocation of the mean shear layer (Fig. 4a,c). The contribution to the overall $\overline{T K E}$ of the different POD modes is changed, and the FVM, FSM, SVM and SSM account for approximately $11.6 \%, 12.9 \%, 4.9 \%$ and $4.2 \%$ at $\mathrm{M}_{\text {ref }}=0.60$ while no notable changes were observed at $\mathrm{M}_{\text {ref }}=0.27$. The overall contribution of the identified POD modes to the overall $\overline{T K E}$ was approximately $35 \%$ at $\mathrm{M}_{\text {ref }}=0.60$ and 0.27 respectively. At $\mathrm{M}_{\mathrm{ref}}=0.60$, the PS of the FVM temporal coefficient $a_{F V M}$ (Fig. 11a) shows significant contributions from $\mathrm{St}=0.26-1.0$. The $\mathrm{SVM}$ shows a minor PS peak at approximately $\mathrm{St}=0.9$ (Fig. 11c). The PS of the FSM (Fig. 11b) shows a dominant frequency at $\mathrm{St}=0.32$, which is also shared by the $\mathrm{SSM}$ (Fig. 11d). Similar coherent structures were obtained at $\mathrm{M}_{\mathrm{ref}}=0.27$, which show similar PS as for the $\mathrm{M}_{\mathrm{ref}}=0.60$ case. For example, the dominant frequency of the FSM and SSM was found at approximately $\mathrm{St}=0.32$ at $\mathrm{M}_{\mathrm{ref}}=0.60$ (Fig. $11 \mathrm{~b}, \mathrm{~d}$ ), and $\mathrm{St}=0.33$ at $\mathrm{M}_{\mathrm{ref}}=0.27$. Therefore, it is concluded that the inlet Mach number has only a minor effect on the spatial distribution and frequency of the main coherent structures of this flow. 
Page 23 of $\mathbf{3 6}$

High-offset $(\mathrm{H} / \mathrm{L}=0.49), \mathrm{M}_{\text {ref }}=0.60$

Out-of-plane velocity

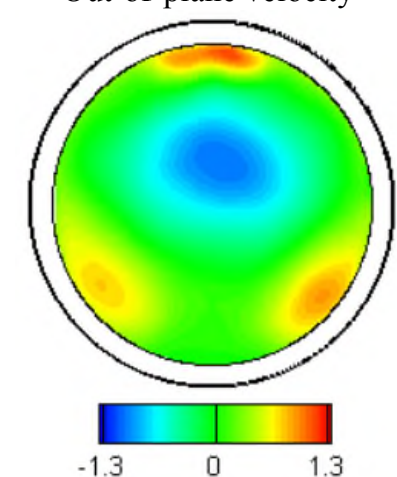

(a) $\Phi_{F V M}^{W}$

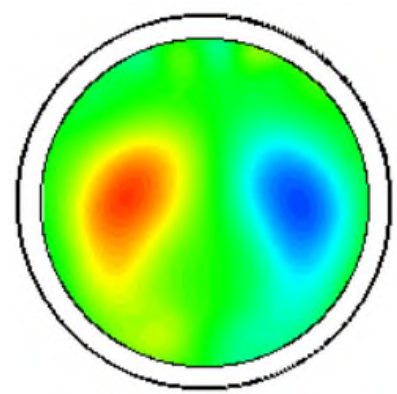

(c) $\Phi_{F S M}^{w}$

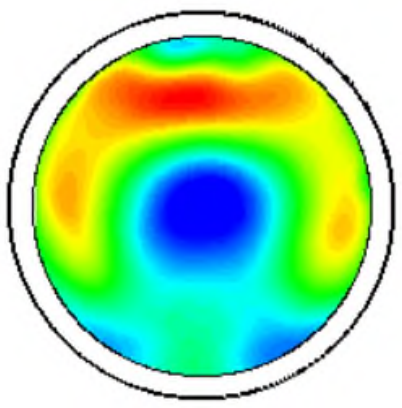

(e) $\Phi_{S V M}^{W}$

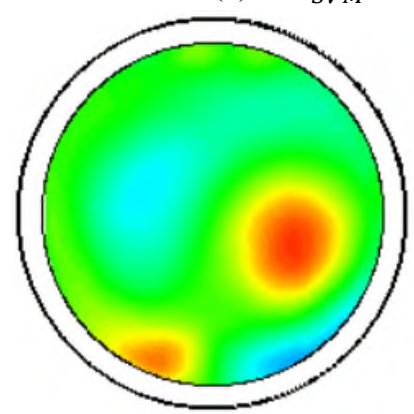

(g) $\Phi_{S S M}^{w}$

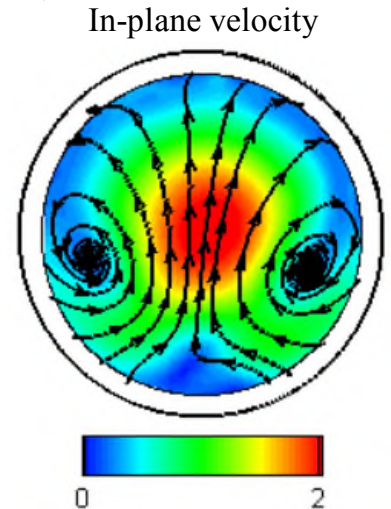

(b) $\Phi_{F V M}^{V_{i p}}$

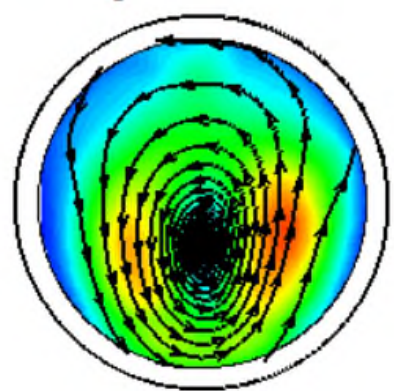

(d) $\Phi_{F S M}^{V_{i p}}$

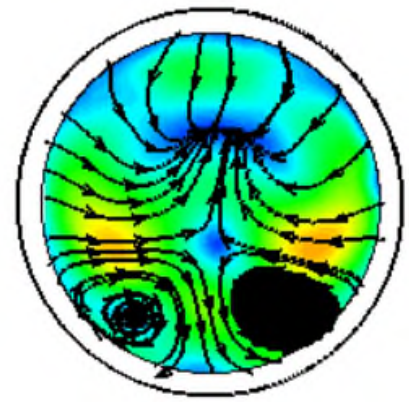

(f) $\Phi_{S V M}^{V_{i p}}$

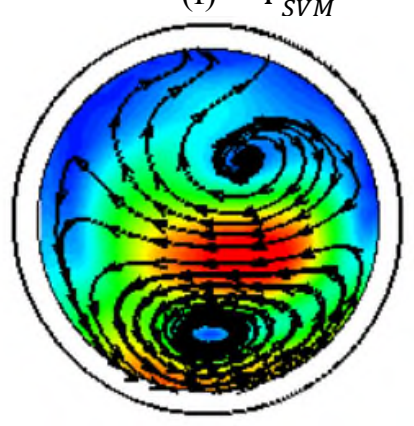

(h) $\Phi_{S S M}^{V_{i p}}$

Fig. 8 Main normalized POD modes of the AIP velocity field, high-offset S-duct $(\mathrm{H} / \mathrm{L}=\mathbf{0 . 4 9})$ at $\mathbf{M}_{\text {ref }}=0.60$. 
Page 24 of 36

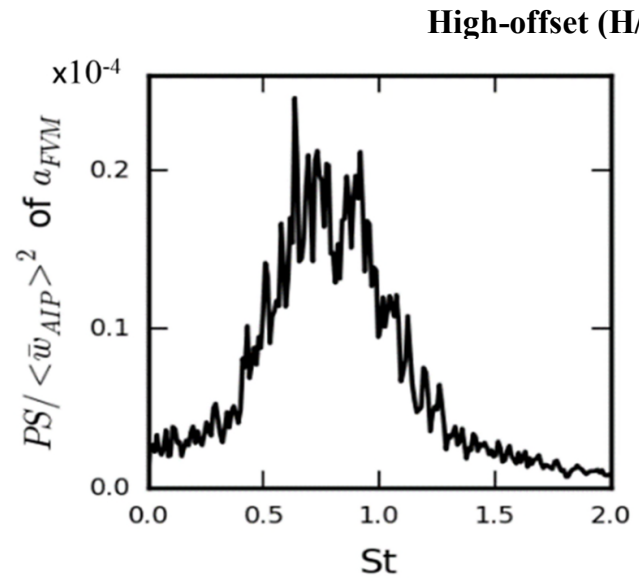

(a)

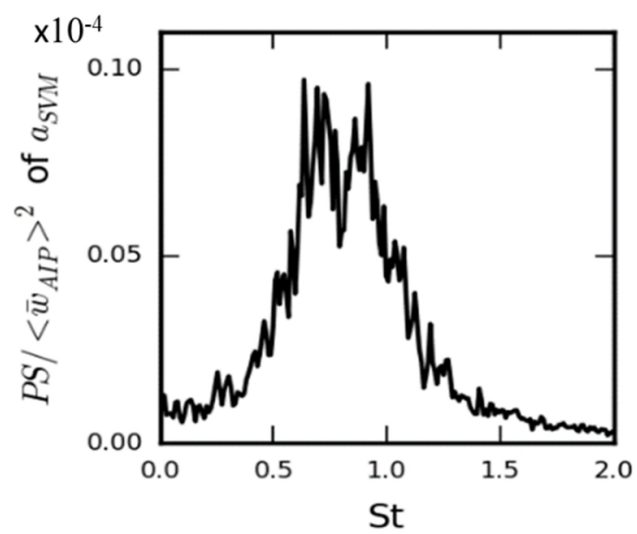

(c)

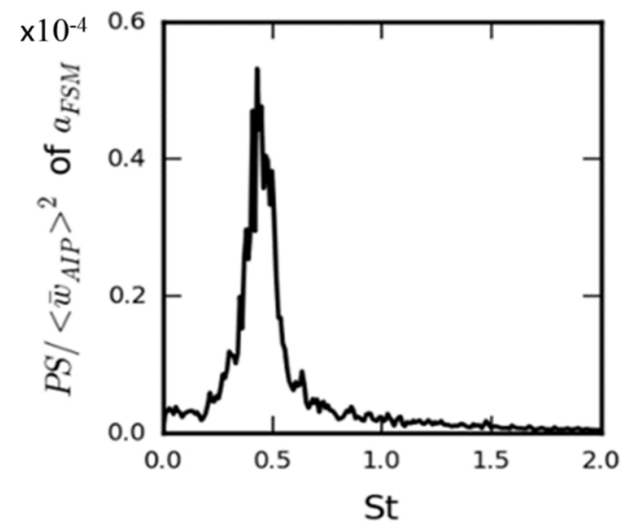

(b)

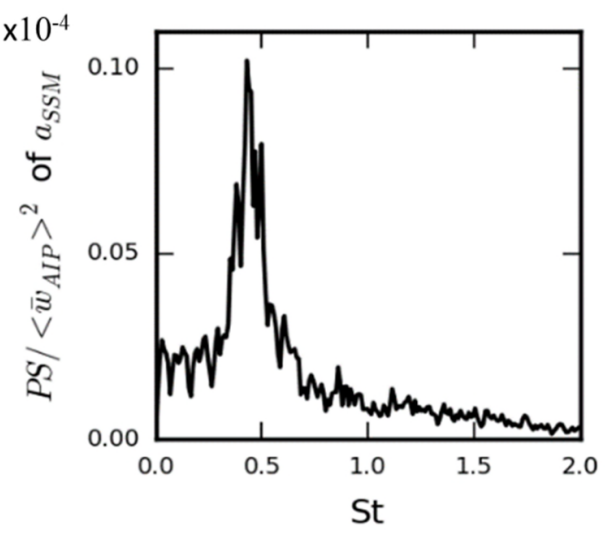

(d)

Fig. 9 Power spectrum of the temporal coefficients of the main AIP velocity POD modes, high-offset S-duct $(\mathrm{H} / \mathrm{L}=0.49)$ at $\mathrm{M}_{\mathrm{ref}}=\mathbf{0 . 6 0}$. 
Page $\mathbf{2 5}$ of $\mathbf{3 6}$

Low-offset $(H / L=0.27), M_{\text {ref }}=0.60$

Out-of-plane velocity

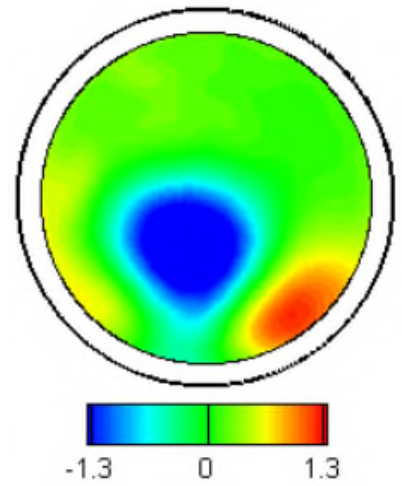

(a) $\Phi_{F V M}^{W}$

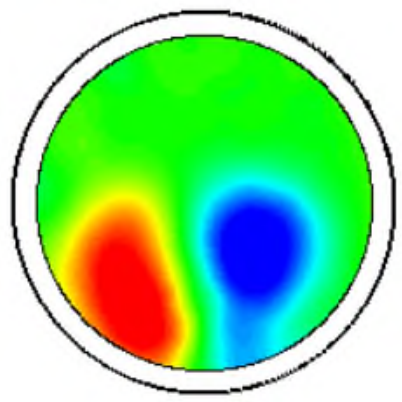

(c) $\Phi_{F S M}^{W}$

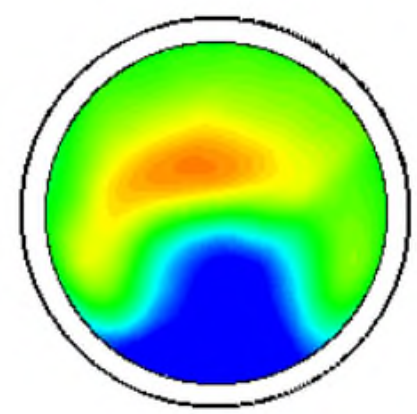

(e) $\Phi_{S V M}^{W}$

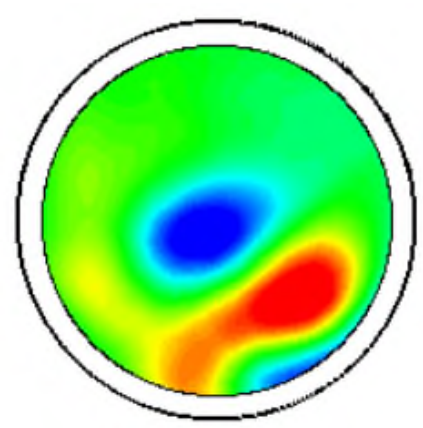

(g) $\Phi_{S S M}^{w}$

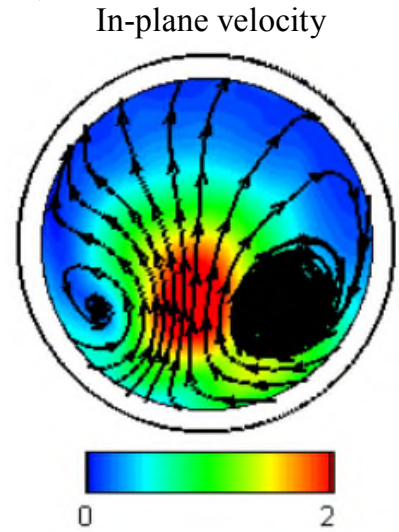

(b) $\Phi_{F V M}^{V_{i p}}$

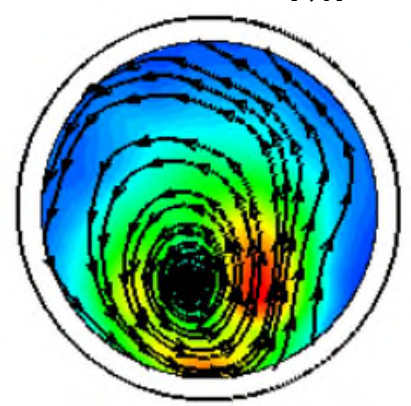

(d) $\Phi_{F S M}^{V_{i p}}$

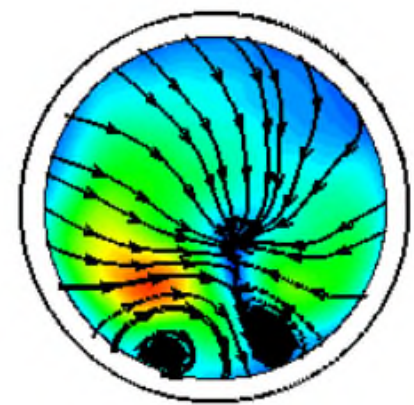

(f) $\Phi_{S V M}^{V_{i p}}$

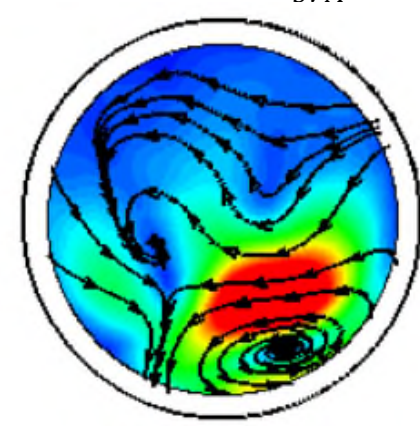

(h) $\Phi_{S S M}^{V_{i p}}$

Fig. 10 Main normalized POD modes of the AIP velocity field, low-offset S-duct $(H / L=0.27)$ at $M_{\text {ref }}=0.60$. 
Page 26 of 36

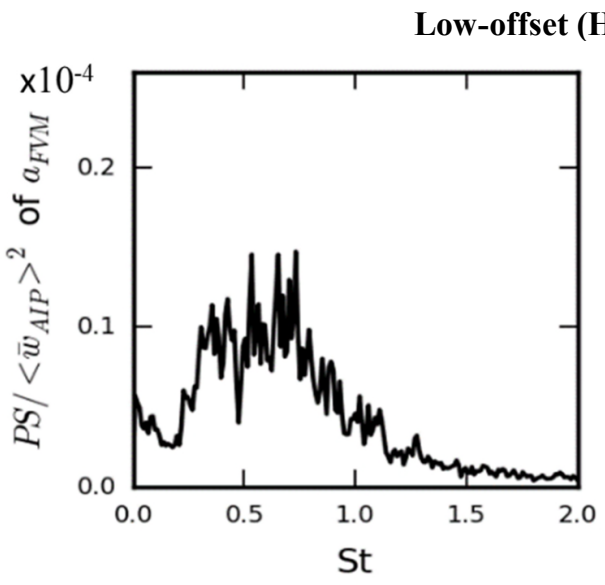

(a)

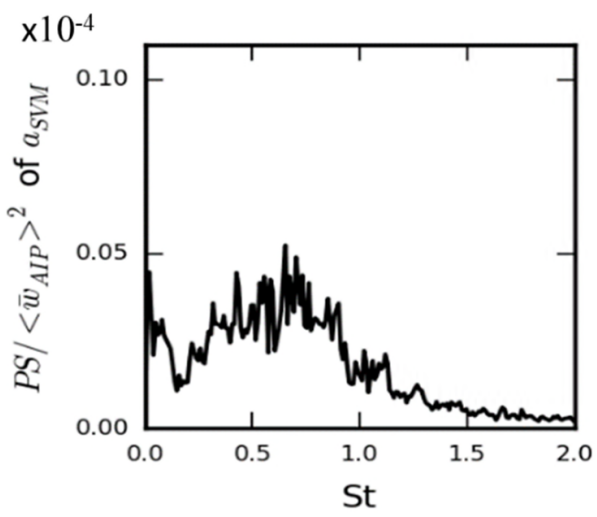

(c)

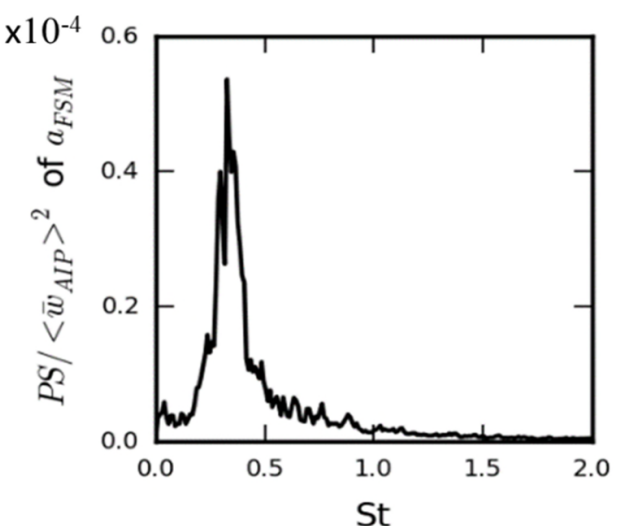

(b)

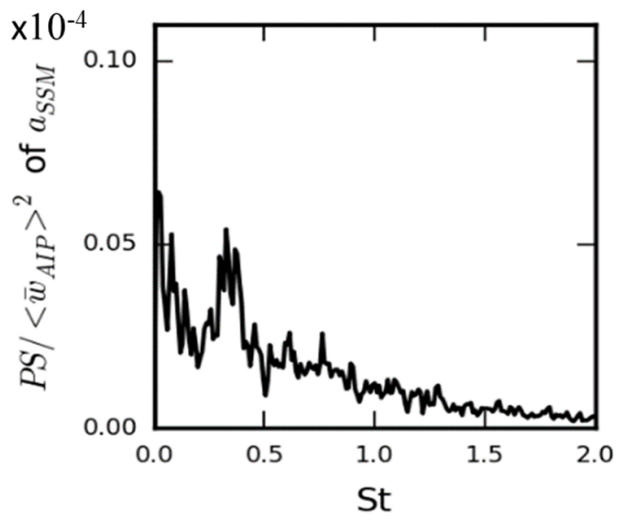

(d)

Fig. 11 Power spectrum of the temporal coefficients of the main AIP velocity POD modes, low-offset S-duct $(\mathrm{H} / \mathrm{L}=0.27)$ at $\mathrm{M}_{\mathrm{ref}}=\mathbf{0 . 6 0}$. 
Page 27 of 36

\subsection{Distortion descriptor unsteady and spectral analysis}

Probability cloud maps between SD and SP distortion descriptors were produced using the TR-PIV data for the two S-duct configurations. These probability maps enable the assessment of the relative likelihood of the various distortion swirl patterns that occur within the flow. The probability maps are generated at $r / R_{\text {AIP }}=0.32$ where the most intense swirl distortions occur for both S-ducts (Gil-Prieto et al. 2017a). Even though the mean flow field shows a steady twin swirl pattern $(\mathrm{SP}=1.0, \mathrm{SD}=0.0)$ with a symmetric, counter-rotating pair of vortices, the probability maps indicate notable deviations from that behavior. For example, positive $(\mathrm{SP}=0.5, \mathrm{SD}=1.0)$ and negative $(\mathrm{SP}=0.5, \mathrm{SD}=$ 1.0) bulk swirl patterns are identified in both configurations at $\mathrm{M}_{\mathrm{ref}}=0.6$, whereby the flow rotates in a single direction (Fig. 12a,b). At $r / R_{A I P}=0.32$, the SP-SD joint-PDF for the high-offset $S-d u c t ~(H / L=0.49)$ at $M_{\text {ref }}=0.6$ shows a tri-modal behavior whereby the flow oscillates between three high-probability areas associated with twin $(\mathrm{SP}=1.0, \mathrm{SD}=0.0)$, positive bulk $(\mathrm{SP}=0.5, \mathrm{SD}=1.0)$ or negative bulk $(\mathrm{SP}=0.5, \mathrm{SD}=-1.0)$ swirl patterns (Fig. 12a). The distorted flow oscillates between these three states and exhibits a wide range of swirl patterns as shown by the SP scatter with values as high as 2.5 (Fig. 12a). It is also possible to identify a high-probability locus between the three dominant states which follows the formula $\mathrm{SP}=1 /(1+|\mathrm{SD}|)$ (Fig. 3). This twin-to-bulk swirl alternating flow behavior represents the lowest value that SP can obtain for any given SD, and holds only for a one-per-revolution swirl pattern with just two swirling regions (Fig. 12a). Along this dominant path, one of the two swirling regions becomes alternately dominant while the other one disappears and the flow switches between positive and negative swirl patterns with a symmetric twin structure amid the two extreme states. Any deviation from this trajectory towards greater SP values indicates the presence of additional swirling regions in this ring. In the low-offset configuration $(\mathrm{H} / \mathrm{L}=0.27)$ at $\mathrm{M}_{\mathrm{ref}}=0.60$ the jointPDF map indicates a more dominant twin swirl pattern and relatively less frequent excursions towards bulk swirl events (Fig. 12b). This oscillating flow behavior is also reflected on the range of the unsteady swirl intensity descriptor SI cloud maps, which indicate notable fluctuations of the SI across a range between $2.5^{\circ}-25^{\circ}$ in both S-duct configurations (Fig. 12c,d). Although the joint-PDF show that the highest probability events are characterized by an SI of around $5^{\circ}$ in both cases, the range across which the SI extends is almost an order of magnitude greater indicating the importance of unsteady SI assessments in relation to fan stability identification. In general, the low-offset S-duct shows a more uniformly distributed joint-PDF around the dominant twin swirl pattern. Broadly the same behavior was observed for both $\mathrm{S}$-ducts at $\mathrm{M}_{\mathrm{ref}}=0.27$ indicating the weak impact of the inlet Mach number on the unsteady swirl distortion patterns. 
Page 28 of 36

High-offset $(\mathrm{H} / \mathrm{L}=0.49), \mathrm{M}_{\mathrm{ref}}=\mathbf{0 . 6 0}$

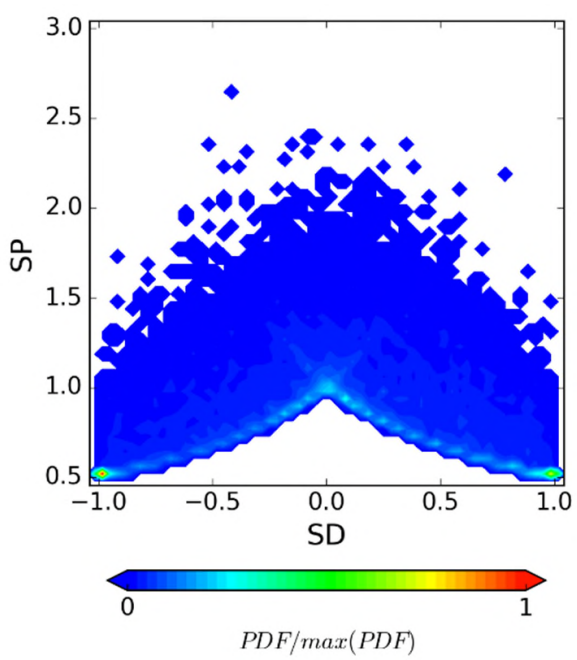

(a)

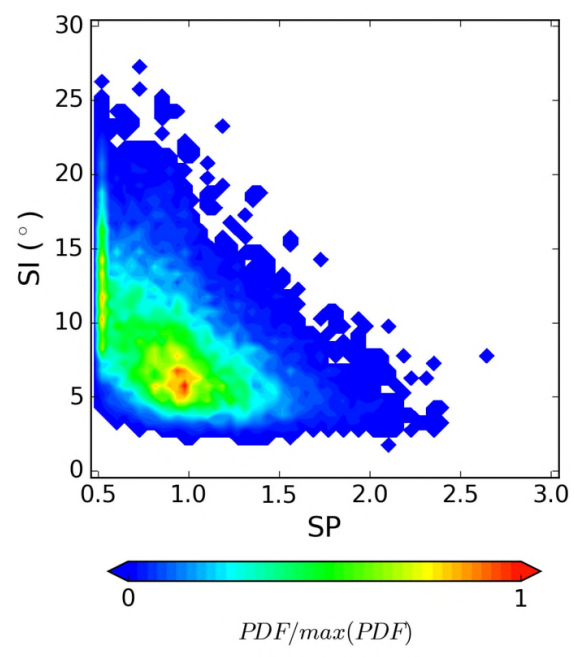

(c)
Low-offset $(\mathrm{H} / \mathrm{L}=\mathbf{0 . 2 7}), \mathrm{M}_{\text {ref }}=\mathbf{0 . 6 0}$

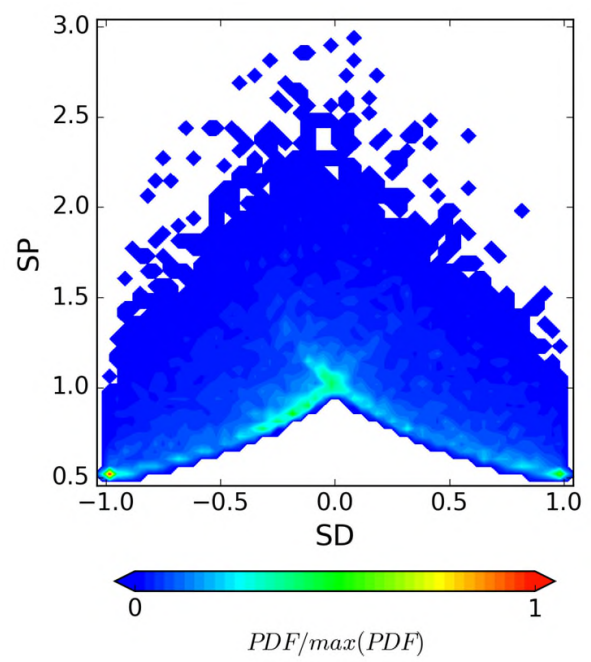

(b)

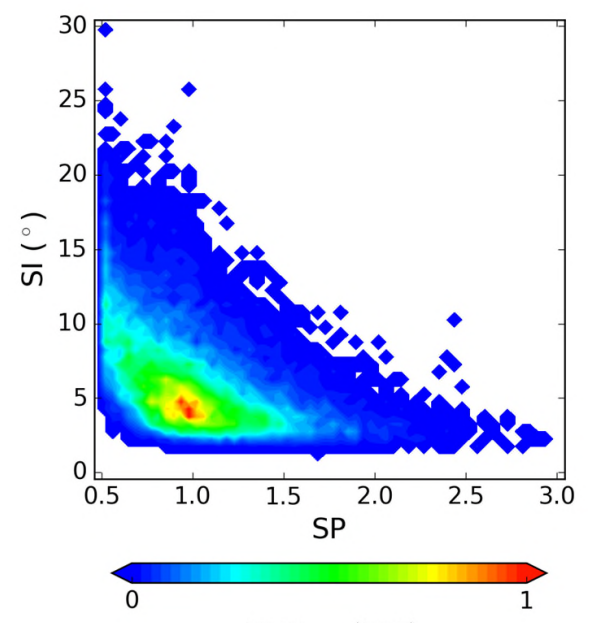

$P D F / \max (P D F)$

(d)

Fig. 12 SP-SD and SI-SP probability maps at $r / R_{\mathrm{AIP}}=0.32$ at $\mathrm{M}_{\mathrm{ref}}=0.60$. 


\section{Page 29 of 36}

The TR-SPIV data also enable spectral analyses of the swirl distortion descriptors for swirl directivity $S D_{i}$ and swirl intensity $S I_{i}$ which are used to characterize the distortion patterns at the AIP. Significant fluctuations in distortion would affect the engine stability if the associated frequencies are within the critical frequency range of a fan (Cousins 2004). As such, the frequency content of the distortion descriptor signals as measured in this work is of key importance to better understand the potential impact on a propulsion system located downstream of the intake. For the purposes of this study the descriptors are evaluated at four radial positions $i=1,2,3,4$ which correspond to $r / R_{A I P}=0.32,0.55$, 0.71 and 0.84 respectively. At each radial position 72 equi-spaced azimuthal positions were considered. The power spectra have been non-dimensionalised with the variance of the corresponding time signal, and therefore the value of the non-dimensional power spectrum at each frequency represents the contribution to the overall variance.

For the high-offset S-duct configuration $(\mathrm{H} / \mathrm{L}=0.49)$ at $\mathrm{M}_{\mathrm{ref}}=0.60$, the swirl directivity $S D i$ shows a distinct dominant frequency at approximately $\mathrm{St}=0.42$ across the range of radial positions considered Fig. 13a,b,c,d). This frequency coincides with the associated frequency of the switching mechanisms previously identified, which indicates that this particular flow feature governs the oscillations of the swirl directivity between positive $(\mathrm{SD}=1)$ and negative $(\mathrm{SD}=-1)$ bulk swirl events. The swirl intensity SIi shows a more broadband spectrum for all radii considered, with the most notable within the range $\mathrm{St}<1.2$ and a significant cut off at $\mathrm{St}>1.5$ (Fig. 13e,f,g,h).

For the low-offset S-duct with $\mathrm{H} / \mathrm{L}=0.27$ at $\mathrm{M}_{\mathrm{ref}}=0.60$ the swirl directivity also appears to be governed by the switching mechanism as it shows a distinct frequency at $\mathrm{St}=0.32$ at all radii except the inner-most one (Fig. 14a,b,c,d). Note that at the inner-most radial position $\mathrm{r} / \mathrm{RAIP}=0.32$ the spectrum of the $S D i$ is significantly more broadband and shows no distinct frequencies (Fig. 14a). The main contributions of the swirl intensity $\left(S I_{i}\right)$ signal across all radii are contained within approximately $\mathrm{St}<1.0$ and a noticeable cut-off occurs at higher frequencies (Fig. 14e,f,g,h). The swirl descriptor behavior was found to be similar at the low inlet Mach number data $\left(\mathrm{M}_{\mathrm{ref}}=0.27\right)$ confirming the weak impact of the inlet Mach number on the unsteady aerodynamics of the two S-duct considered. 
Page $\mathbf{3 0}$ of $\mathbf{3 6}$

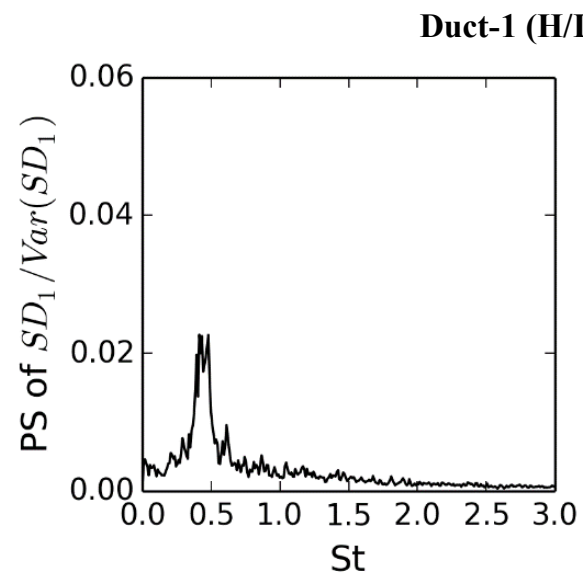

(a)

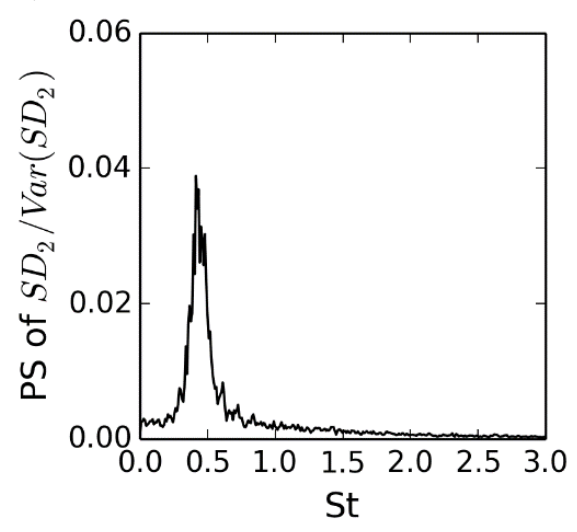

(b)
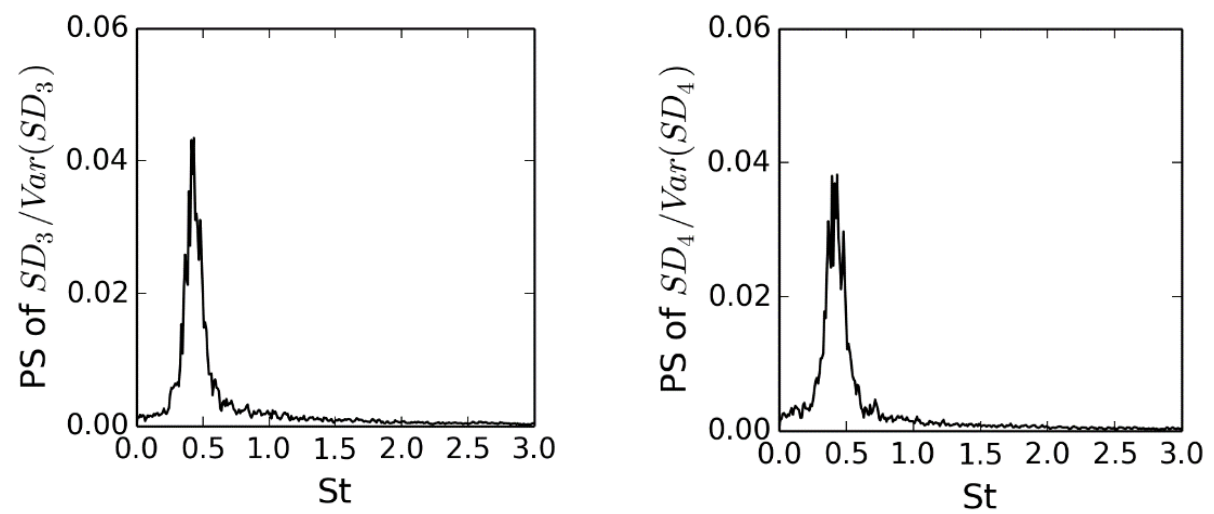

(c)

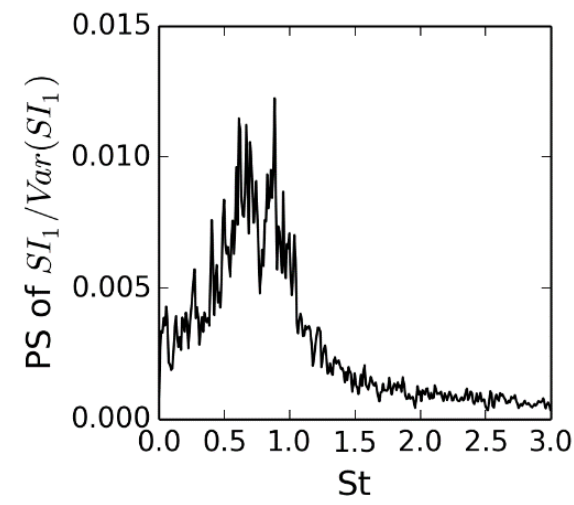

(e)

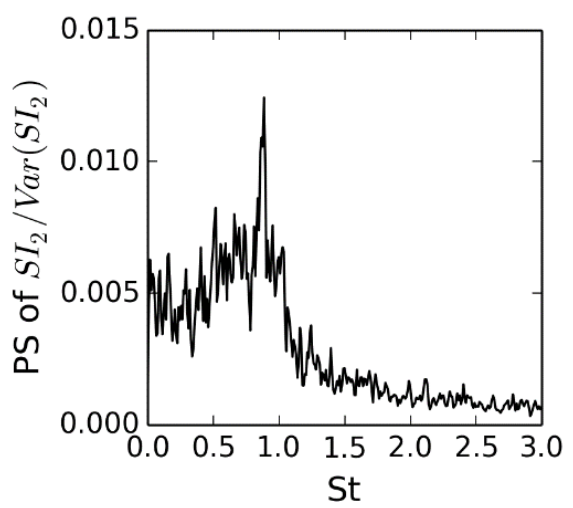

(f) 
Page 31 of 36

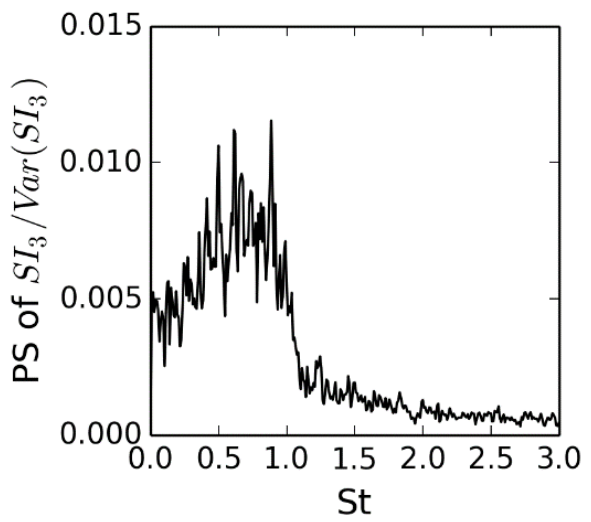

(g)

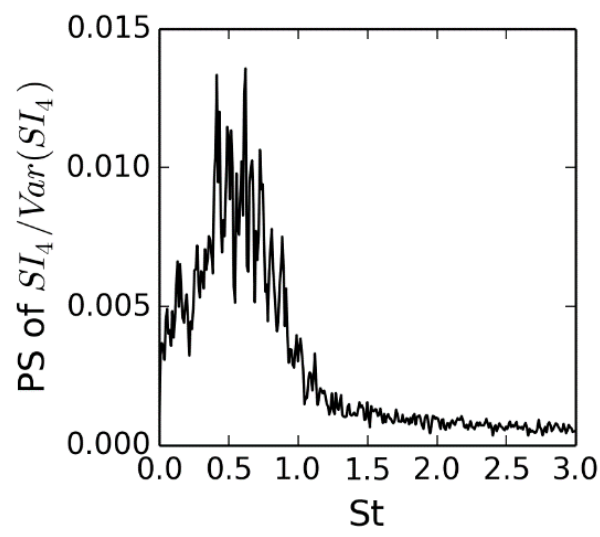

(h)

Fig. 13 Power spectrum of the $S D_{i}$ and $S I_{i}$ swirl distortion metrics across a range of radial positions with $i$

$=1,2,3,4$ correspond to $r / R_{\text {AIP }}=0.32,0.55,0.71,0.84$. The power spectrum is non-dimensionalised by the total variance of the corresponding time-signal. High-offset $S-d u c t(H / L=0.49)$ at $M_{\text {ref }}=0.60$.

Duct-2 $(\mathrm{H} / \mathrm{L}=\mathbf{0 . 2 7}), \mathrm{M}_{\mathrm{ref}}=\mathbf{0 . 6 0}$

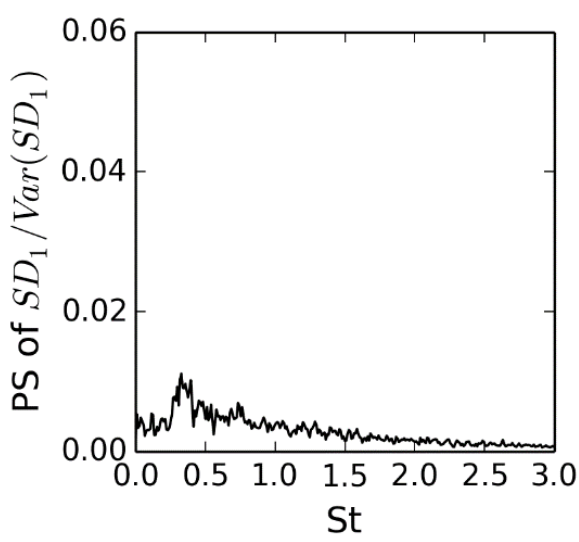

(a)

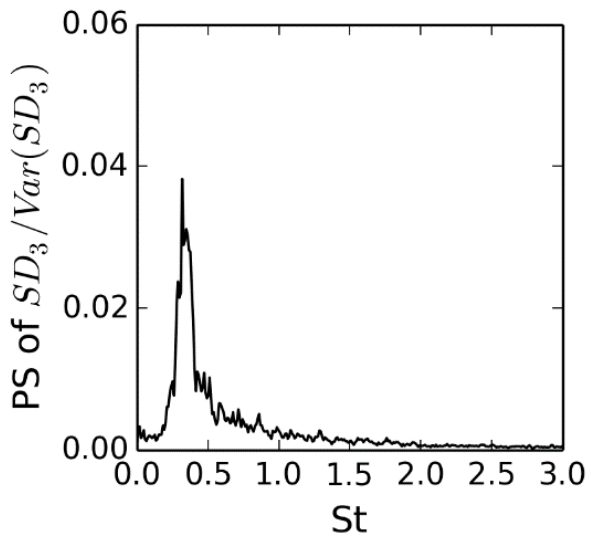

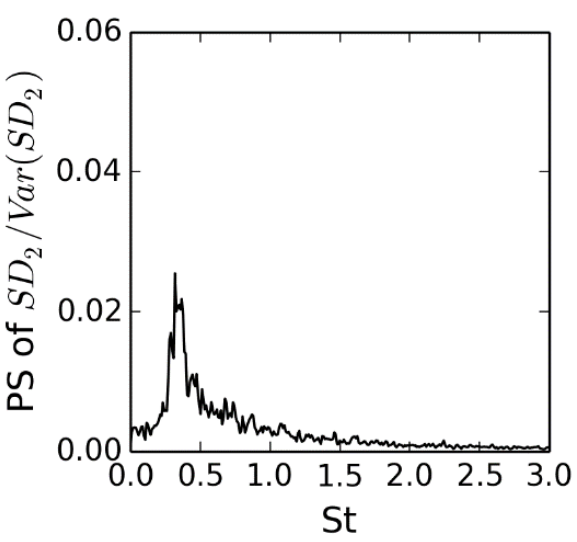

(b)

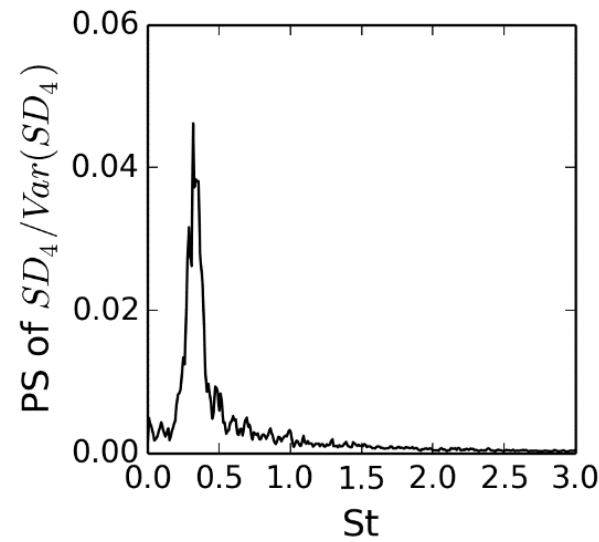


Page 32 of 36

(c)

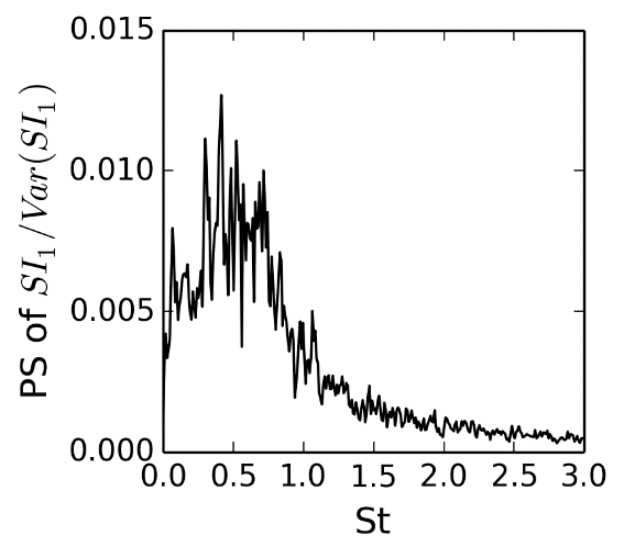

(e)

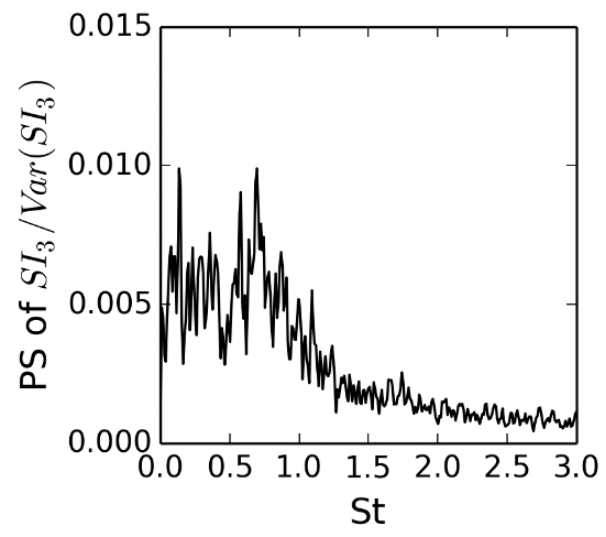

(g) (d)

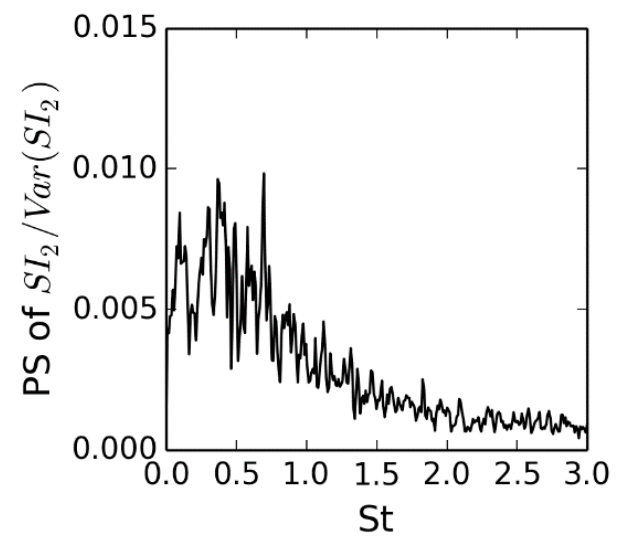

(f)

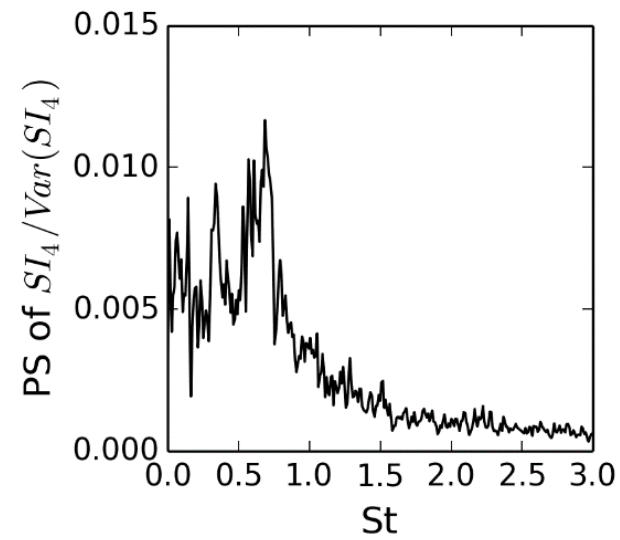

(h)

Fig. 14 Power spectrum of the $S D_{i}$ and $S I_{i}$ Swirl distortion metrics across a range of radial positions with $i$

$=1,2,3,4$ correspond to $r / R_{\text {AIP }}=0.32,0.55,0.71,0.84$. The power spectrum is non-dimensionalised by the total variance of the corresponding time-signal. Low-offset $S-d u c t(H / L=0.27)$ at $M_{\text {ref }}=0.60$. 
Page 33 of 36

\section{Conclusions}

This work shows the first application of TR-SPIV for the measurement of the distorted, three-component velocity field at the AIP of convoluted aero-engine intakes with high spatial and temporal resolution. Compared to conventional unsteady total-pressure measurements, TR-PIV provides about 45 times more measurement points, and enables the synchronous assessment of the in-plane and out-of-plane flow features in the frequency domain. These characteristics make TR-SPIV an attractive approach to quantify and characterize these complex flow fields.

The main frequencies of the flow field within the two intake configurations were found to be lower than $\mathrm{St}=1.2$, and therefore within the estimated critical frequency range of $\mathrm{St}=0.9-5.4$ to which a typical aero-engine fan would respond. Therefore, the associated perturbations can potentially have a destabilizing effect on the engine. The spectral and POD analyses showed that the main source of unsteadiness was the swirl switching mechanism. The swirl switching comprises the alternate dominance of one of the AIP Dean vortices, with a concomitant lateral oscillation of the main loss region, which follows the movement of the dominant vortex. The oscillation of the main loss region and the switching between the clockwise and counter-clockwise vortices occurs at a fundamental frequency of $\mathrm{St}=0.42$ and 0.32 for the high and low offset ducts at $\mathrm{M}_{\mathrm{ref}}=0.60$. This is also reflected in the $S D_{i}$ spectrograms of both ducts which indicate that the swirl directivity oscillations are driven by these flow features. Another significant source of unsteadiness for both ducts was a vertical oscillation of the main loss region around the center of the AIP, promoted by the unsteadiness of the shear layer and associated with a more broadband spectrum. For the low offset duct at $\mathrm{M}_{\mathrm{ref}}=0.60$, important contributions to this flow feature were observed between $\mathrm{St}=0.26-1.0$. For the high offset duct at $\mathrm{M}_{\mathrm{ref}}=0.60$, this vertical perturbation showed contributions mainly between $\mathrm{St}=0.6$ and 1.0. These fundamental frequencies of the velocity field were also reflected on the swirl intensity $\left(S I_{i}\right)$ temporal behavior, which represents an indicator of potential engine instabilities. For example, the unsteady maximum levels of swirl distortion were found to be approximately 5-times greater than the time-averaged values. The inlet Mach number had only a minor impact on the flow field spectrum and main flow features across the investigated range between $\mathrm{M}_{\mathrm{in}}=0.27$ and 0.6 , which is a representative operating regime for a subsonic intake.

Overall, the main frequencies and the associated coherent structures of the distorted flow field in two S-shaped intakes are quantified. The effect of the S-duct centerline offset and Mach number on the unsteadiness distribution and spectral content were shown. Compared with conventional unsteady total-pressure measurements, TR-PIV offers 
Page $\mathbf{3 4}$ of $\mathbf{3 6}$

a greater spatial resolution and the possibility to discern between in- and out-of-plane flow features, which is of major importance to understand the unsteady flow field structures that are delivered to the aero-engine fan. 
Page 35 of 36

\section{References}

Anderson BH, Reddy DR, Kapoor K (1994) Study on Computing Separating Flows within a Diffusion Inlet S-duct. J. of Propulsion and Power, Vol. 10, No. 5, pp. 661-667. Doi: 10.2514/3.23777

Berens TM, Delot AL, Chevalier M, Van Muijden J (2014) Numerical Simulations for High Offset Intake Diffuser Flows. 52nd AIAA Aerospace Sciences Meeting - AIAA SciTech 2014, AIAA 2014-0371, National Harbor, Maryland, USA. Doi: $10.2514 / 6.2014-0371$

Berkooz G, Holmes P, Lumley JL (1993) The Proper Orthogonal Decomposition in the Analysis of Turbulent Flows. Annual Review of Fluid Mechanics, Vol. 25, pp. 539-575. Doi: 10.1146/annurev.fl.25.010193.002543

Bowditch DN, Coltrin RE (1983) A Survey of Inlet/Engine Distortion Compatibility. NASA TM-83421.

Breuer T, Bissinger NC (2010) Basic Principles: Gas Turbine Compatibility - Gas Turbine Aspects. Encyclopedia of Aerospace Engineering. Doi: 10.1002/9780470686652.eae573

Cousins WT (2004) History, Philosophy, Physics and Future Directions of Aircraft Propulsion System / Inlet Integration. Proceedings of ASME Turbo Expo 2004: Power for Land, Sea, and Air, Vol. 2, GT2004-54210, pp. 305-320. Doi: $10.1115 /$ GT2004-54210

Crites RC, Heckart MV (1971) Application of Random Data Techniques to Aircraft Inlet Diagnostics. J. Aircraft, Vol. 8, No. 7, pp. 502-510. Doi: 10.2514/3.59130

Dean WR (1927) Note on the Motion of Fluid in a Curved Pipe. The London, Edinburgh, and Dublin Philosophical Magazine and Journal of Science, Vol. 4, No. 20, pp. 208-223. Doi: 10.1080/14786440708564324

Delot A-L, Garnier E, Pagan D (2011) Flow Control in a High-Offset Subsonic Air Intake. 47th AIAA/ASME/SAE/ASEE Joint Propulsion Conference \& Exhibit, AIAA 2011-5569, San Diego, California. Doi: 10.2514/6.2011-5569

Fiola C, Agarwal RK (2015) Simulation of Secondary and Separated Flow in Diffusing S-ducts. J of Propulsion and Power, Vol. 31, No. 1, pp. 180-191. Doi: 10.2514/1.B35275

Florea RV, Matalanis C, Hardin LW, Stucky M, Shabbir A (2015) Parametric Analysis and Design for Embedded Engine Inlets. Journal of Propulsion and Power, Vol. 31, No. 3, pp. 843-850. Doi: 10.2514/1.B34804

Gan G, Zhang X (2017) Design Optimization of a Three-dimensional Diffusing S-duct Using a Modified SST Turbulence Model. Aerospace Science and Technology, Vol. 63, pp. 63-72. Doi: 10.1016/j.ast.2016.12.016

Garnier E (2015) Flow Control by Pulsed Jet in a Curved S-Duct: A Spectral Analysis. AIAA Journal, Vol. 53, No. 10, pp. 2813-2827. Doi: 10.2514/1.J053422

Gil-Prieto D, MacManus DG, Zachos PK, Tanguy G, Menzies KR (2017) Convoluted Intake Distortion Measurements Using Stereo Particle Image Velocimetry. AIAA Journal, Vol. 55, No. 6, pp. 1878-1892. Doi: 10.2514/1.J055467

Gil-Prieto D, MacManus DG, Zachos PK, Tanguy G, Wilson F, Chiereghin N (2017) Delayed Detached-Eddy Simulation and Particle Image Velocimetry Investigation of S-Duct Flow Distortion. AIAA Journal, Vol. 55, No. 6, pp. 1893-1908. Doi: 10.2514/1.J055468

Gil-Prieto D, MacManus DG, Zachos PK, Bautista A (2018) Assessment Methods for Unsteady Flow Distortion in Aeroengine Intakes. Aerospace Science and Technology, Vol. 72, pp. 292-304. Doi: 10.1016/j.ast.2017.10.029

Gissen AN, Vukasinovic B, Glezer A (2014) Dynamics of flow control in an emulated boundary layer-ingesting offset diffuser. Experiments in Fluids, Vol. 55:1794, pp. 1-19. Doi: 10.1007/s00348-014-1794-5

Hellström LHO, Zlatinov MB, Cao G, Smits AJ (2013) Turbulent Pipe Flow Downstream of a $90^{\circ}$ Bend. Journal of Fluid Mechanics, Vol. 735, R7, pp. 1-12. Doi: 10.1017/jfm.2013.534

Kalpakli Vester A, Örlü R, Alfredsson PH (2015) POD analysis of the turbulent flow downstream a mild and sharp bend. Experiments in Fluids, Vol. 56, No. 3, Article id. 57. Doi: 10.1007/s00348-015-1926-6

Kawai RT, Friedman DM, Serrano L (2006) Blended Wing Body (BWB) Boundary Layer Ingestion (BLI) Inlet Configuration and System Studies. NASA Technical Report CR-2006-214534.

Kim H, Liou M-S (2013) Shape Design Optimization of Embedded Engine Inlets for N2B Hybrid Wind-body Configuration. Aerospace Science and Technology, Vol. 30, pp. 128-149. Doi: 10.1016/j.ast.2013.07.011

Knoth F, Breitsampter C (2017) Flow Analysis of a Helicopter Engine Side Air Intake. J. of Propulsion and Power, Vol. 33, No. 5, pp. 1230-1244. Doi: 10.2514/1.B36285 


\section{Page 36 of 36}

Kostas J, Soria J, Chong MS (2002) Particle image velocimetry measurements of a backward-facing step flow. Experiments in Fluids, Vol. 33, No. 6, pp. 838-853. Doi: 10.1007/s00348-002-0521-9

Kostas J, Soria J, Chong MS (2005) A comparison between snapshot POD analysis of PIV velocity and vorticity data. Experiments in Fluids, Vol. 38, No. 2, pp. 146-160. Doi: 10.1007/s00348-004-0873-4

Lee, DT, Schachter, BJ (1980) Two algorithms for constructing a Delaunay triangulation. International Journal of Computer and Information Sciences Vol. 9, No. 3, pp 219-242. https://doi.org/10.1007/BF00977785

Lumley JL (2007) Stochastic Tools in Turbulence. Dover ed., Dover Publications, Inc., New York, USA, Chap. 3, pp. 57-59.

MacManus DG, Chiereghin N, Gil-Prieto D, Zachos P (2017) Complex Aeroengine Intake Ducts and Dynamic Distortion. AIAA Journal. Doi: 10.2514/1.J054905

Paul DL, Younghans JL (1978) Chapter 13 - Inlets and Inlet Engine Integration. The Aerothermodynamics of Aircraft Gas Turbine Engines, edited by Oates, G. C., Report AFAPL TR 78-52, University of Washington Seattle.

Raffel M, Willert C, Wereley S, Kompenhans J (2007) Particle Image Velocimetry: A Practical Guide. $2^{\text {nd }}$ ed., Springer-Verlag, Berlin, Chap. 5, pp. 164-176. Doi: 10.1007/978-3-540-72308-0

Society of Automotive Engineers (2007) A Methodology for Assessing Inlet Swirl Distortion. SAE Aerospace Information Report 5686, Warrendale, PA, USA.

Strazisar AJ, Wood JR, Hathaway MD, Suder KL (1989) Laser Anemometer Measurements in a Transonic Axial-Flow Fan Rotor. NASA Technical Paper 2879.

Tanguy G, MacManus DG, Garnier E, Martin PG (2018) Characteristics of Unsteady Total Pressure Distortion for a Complex Aero-engine Intake. Aerospace Science and Technology, Vol. 78, pp. 297-311. Doi: 10.1016/j.ast.2018.04.031

Taylor JR (1997) An Introduction to Error Analysis. The study of uncertainties in physical measurements, $2^{\text {nd }}$ ed., University Science Books, Sausalito, CA, USA, Chap. 3, pp. 73-75.

Toge TD, Pradeer AM (2017) Experimental Investigation of Stall Inception of a Low Speed Contra Rotating Axial Flow Fan under Circumferential Distorted Flow Condition. Aerospace Science and Technology, Vol. 70, pp. 534-548. Doi: 10.1016/j.ast.2017.08.041

Tunstall MJ, Harvey JK (1968) On the effect of a sharp bend in a fully developed turbulent pipe-flow. Journal of Fluid Mechanics, Vol. 34, No. 3, pp. 595-608. Doi: 10.1017/S0022112068002107

Welch PD (1967) The Use of Fast Fourier Transform for the Estimation of Power Spectra: A Method Based on Time Averaging Over Short, Modified Periodograms. IEEE Transactions on Audio and Electroacoustics, Vol. 15, No. 2, pp. 70-73. Doi: 10.1109/TAU.1967.1161901

Wellborn SR, Reichert BA, Okiishi TH (1994) Study of the Compressible Flow in a Diffusing S-Duct. AIAA Journal of Propulsion and Power, Vol. 10, No. 5, pp. 668-675. Doi: 10.2514/3.23778

Wenzel LM, Blaha RJ (1977) Analysis of Dynamic Inlet Distortion Applied to a Parallel Compressor Model. NASA TM X3522 .

Wieneke B (2005) Stereo-PIV using self-calibration on particle images. Experiments in Fluids, Vol. 39, No. 2, pp. 267-280. Doi:10.1007/s00348-005-0962-z

Zachos PK, MacManus DG, Gil-Prieto D, Chiereghin N (2016) Flow Distortion Measurements in Convoluted Aeroengine Intakes. AIAA Journal, Vol. 54, No. 9, pp. 2819-2832. Doi: 10.2514/1.J054904 
2018-10-18

\section{Unsteady characteristics of S-duct intake flow distortion}

Gil-Prieto, Daniel

Elsevier

Gil-Prieto D, Zachos PK, MacManus DG, McLelland G. (2019) Unsteady characteristics of S-duct intake flow distortion. Aerospace Science and Technology, Volume 84, January 2019, pp. 938-952

https://doi.org/10.1016/j.ast.2018.10.020

Downloaded from Cranfield Library Services E-Repository 